\title{
A geometry-dependent model for void closure in hot metal forming processes
}

\author{
M. Saby ${ }^{\mathrm{a}}$, P.-O. Bouchard ${ }^{\mathrm{a}}$, M. Bernacki ${ }^{\mathrm{a}}$ \\ ${ }^{a}$ Mines Paris Tech, CEMEF - Centre de Mise en Forme des Matériaux, CNRS UMR 7635 CS 10207 \\ 1 rue Claude Daunesse, 06904 Sophia Antipolis cedex, France
}

\begin{abstract}
During production of large metal workpieces, an internal presence of voids is usually observed. Such internal defects are generally closed up using hot metal forming processes, such as hot forging or hot rolling. Prediction models for void closure, associated with process simulation, are extremely powerful tools and might significantly support process design. However, there is at present a lack of accurate models being able to predict void closure according to industrial conditions, particularly in terms of void geometries. In this paper, an original model for void closure is presented, accounting for the void's geometry and orientation, as well as the mechanical state during deformation. The model was build and calibrated based on a wide campaign of finite element simulations at the scale of a representative volume element. Various void geometries were defined and several mechanical states were prescribed on a range that is representative of industrial loadings. The model's accuracy was verified using industrial data and was compared to literature. Great advantages were obtained for non-spherical voids in terms of void volume evolution.
\end{abstract}

Keywords: Void closure; Model; Finite elements; Hot metal forming

\section{Introduction}

Industrial needs for large metal components used in aerospace, transport or energy applications constantly increase. During the first steps of elaboration of ingots or preforms, defects, such as voids and internal cavities, may occur. An elimination of these internal defects is required to avoid catastrophic failure during process, or during service of final components. Void elimination is usually performed by means of hot metal forming processes, inducing large deformation in the material at high temperature, and leading to closure of internal voids. Optimization and control of such forming processes in terms of costs and final material soundness remains of prime importance.

The phenomenon of void closure is generally described using two stages: the mechanical closure of void, bringing internal surfaces into contact, and the final bonding of the internal surfaces providing complete healing and thus a sound material (Park and Yang, 1996). The present work focuses on the mechanical closure phenomenon.

Although main studies regarding void closure were published over the last two decades, some qualitative improvements in terms of process conditions were already pointed out using experimental observations in Tomlison et al. (1958) for hot forging, and in Wallerö (1985) for hot rolling. Tomlison et al. studied the effect of hot forging process parameters on void closure and found out that concave

Email address: michel.saby@mines-paristech.fr +33493957415 (M. Saby) 
dies are favourable for void closure. These results were confirmed by Dudra and Im (1990) using FML $^{1}$ dies, by Banaszek and Stefanik (2006) using bowl-shape dies and by Chen et al. (2012) using V-shape dies. For hot rolling processes, Wallerö (1985) pointed out that large roll diameters and large spread passes are recommanded for better void closure. From such results, it was concluded that compressive states have tendency to improve the closure efficiency (Ståhlberg, 1986).

Ståhlberg and Keife (1992) stated that initial cooling of workpieces can be favourable for void closure in the case of hot forging. It is explained by the fact that the temperature gradient induces more compressive stress states in the bulk of workpieces. Pietrzyk et al. (1995) confirmed the favourable effect of pre-cooling workpieces in hot rolling. Överstam and Jarl (2004) also pointed out a coupled effect between friction and temperature regarding their effect on void closure. In fact, all these studies deal with stress states in the workpiece and confirm the favourable effect of compressive states for void closure.

Ståhlberg et al. (1980) studied the deformation of round and square voids in a rigid-perfectly plastic material. They proposed an upper bound for void closure based on the plane-strain condition, considering two different deformation modes around the voids. Tanaka et al. (1986) pointed out that a good indicator for void closure is the integral of stress triaxiality ratio over the cumulated strain. Nakasaki et al. (2006) discussed the use of this variable in the case of hot rolling and proposed a modified model to fit experimental data. Kakimoto et al. (2010) also used the same indicator to establish a criterion for void closure. Based on a series of finite element simulations, the authors concluded that voids are systematically closed when the integral of stress triaxiality reaches a threshold value.

Based on a similar approach, a stress triaxiality based (STB) model for void closure was proposed in the commercial software FORGE ${ }^{\circledR}$ as a post-processing mean-field model (Saby, 2013). This model is able to provide maps of void volume prediction, according to the integral of stress triaxiality ratio over cumulated strain at any position in the workpiece. Calibration of this model can be performed using compression cases of a billet containing a given void (usually spherical), and is therefore limited to the chosen configuration.

Based on the analytical evolution of a sphere found by Duva and Hutchinson (1984) and the analytical evolution of a crack-like shape found by He and Hutchinson (1981), Zhang and Cui (2009) proposed a model for predicting the volume evolution of an initially spherical void, by analytically taking into account the change of shape during deformation. A semi-empirical model was also proposed by the same authors (Zhang et al., 2009), by adding correction terms to the solutions obtained by Duva and Hutchinson (1984), in order to empirically take into account the change of shape during deformation. Although many improvements can be obtained using such models, both works of Zhang and co-workers were restricted to the case of initial spherical voids. Initial non-spherical void shapes may however have a non-negligible impact on void closure rate, such as observed by Lee and Mear (1994) using ellipsoidal voids. In particular, the elongation and orientation of the void with respect to the main compression axis may significantly affect the void closure rate.

This paper presents a new prediction model for void closure, based on a large campaign of numerical simulations at the micro-scale, using a representative volume element (RVE) containing ellipsoidal voids. A description of the RVE simulations is given in the first section. Then a parametric sensitivity study to void geometry (orientation and aspect ratios) and to several material

\footnotetext{
${ }^{1}$ Free of Mannesmann effect at Lower press load
} 
parameters is presented. The parameters that exhibit a first-order influence on void closure are then quantitatively assessed and the prediction model is defined and discussed in the fourth section.

\section{Description of the meso-scale approach}

\subsection{Representative Volume Element}

The mechanisms of void closure are studied using finite element analysis in a tridimensional RVE. This methodology enables the closure mechanisms to be studied at the meso-scale and was presented in previous work (Saby et al., 2013). In the present work, the dimensions of the RVE are $D_{x}=D_{y}=D_{z}=10 \mathrm{~mm}$ and mesh size is set to the value $0.1 \mathrm{~mm}$ around the void in order to ensure validity of the RVE simulations. An ellipsoidal void, with initial dimensions $r_{1}, r_{2}, r_{3}$ (see Fig. 1) is placed at the central position in the RVE. The initial void volume fraction is $f_{0}=10^{-3}$. The initial aspect ratios are defined as the ratios $\frac{r_{3}}{r_{1}}$ and $\frac{r_{2}}{r_{1}}$.

Tridimensional unstructured volume meshing is used, with tetrahedral elements as shown in Fig. 2. The FE simulations are performed using the software FORGE2011 ${ }^{\circledR}$, using a mixed velocitypressure $P 1^{+} / P 1$ formulation.

A visco-plastic behaviour law (Eq.1) is used. This law is known as the Hansel-Spittel law and is widely used for modeling the behaviour of metals at high temperature. The flow stress $\sigma_{0}$ is expressed as:

$$
\sigma_{0}=A\left(\bar{\varepsilon}+\varepsilon_{0}\right)^{n} \dot{\bar{\varepsilon}}^{m} e^{m_{4} /\left(\bar{\varepsilon}+\varepsilon_{0}\right)},
$$

where $A$ is the material consistency at the given temperature (isothermal conditions are assumed here), $m$ the strain-rate sensitivity, $\left(n, m_{4}\right)$ the strain hardening and softening coefficients, and $\varepsilon_{0}$ a regularization term that enables initial rigidity of the material.
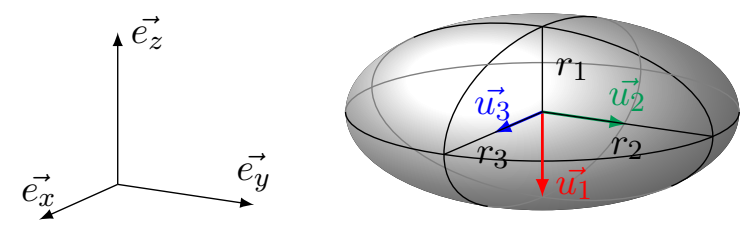

Figure 1: Definition of the void's dimensions and orientation in the canonical basis $\left(\overrightarrow{e_{x}}, \overrightarrow{e_{y}}, \overrightarrow{e_{z}}\right)$.

\subsection{Boundary conditions}

In the large majority of models (e.g. micro-analytical solutions in Duva and Hutchinson (1984), or empirical criteria in Kakimoto et al. (2010)), equivalent strain and stress triaxiality ratio rise as key parameters regarding mechanical influence. Equivalent strain expresses as $\bar{\varepsilon}=\sqrt{\frac{2}{3} \varepsilon: \varepsilon}$ and stress triaxiality ratio $T_{X}=\frac{\sigma_{m}}{\sigma_{e}}$, where $\sigma_{m}=\frac{1}{3} \operatorname{tr}(\boldsymbol{\sigma})$ is the mean stress and $\sigma_{e}=\sqrt{\frac{3}{2} \boldsymbol{\sigma}: \boldsymbol{\sigma}}$ is the von Mises equivalent stress. Void volume evolution is generally presented as a function of equivalent strain $\bar{\varepsilon}$. It was also observed that strain-rate has no influence on void volume evolution (Saby et al., 2013). Consequently, an arbitrary constant strain-rate is applied with the value $\dot{\bar{\varepsilon}}=1 \mathrm{~s}^{-1}$. The constant strain-rate is prescribed using a normal velocity $V_{z}(t)=-D_{z}(t)$ on the upper surface of the RVE, i.e. along the $z$-axis (see Fig. 3).

In this work, it is chosen to prescribe a constant stress triaxiality ratio in order to quantitatively study its influence on void closure. Normal stresses are applied along the $x$ and $y$-axes using 


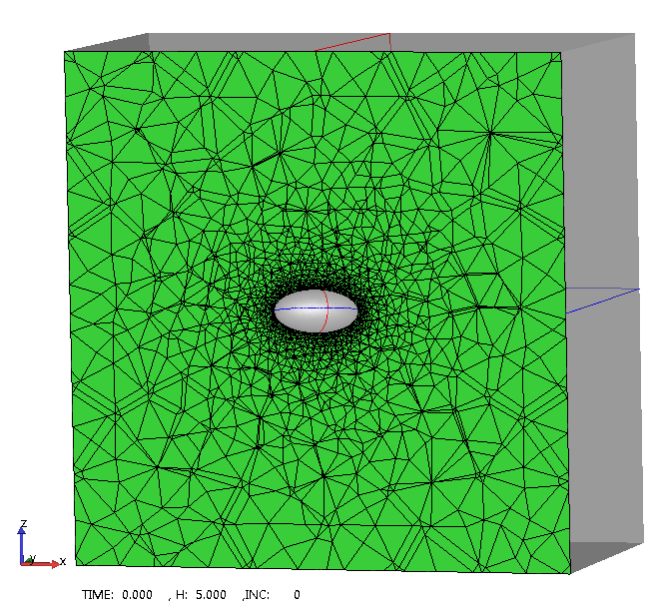

(a)

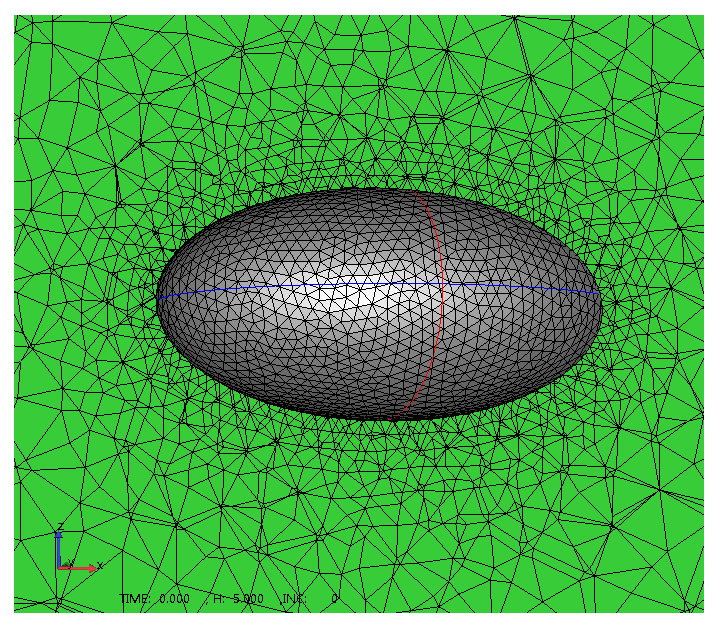

(b)

Figure 2: Initial mesh of the RVE containing an ellipsoidal void.

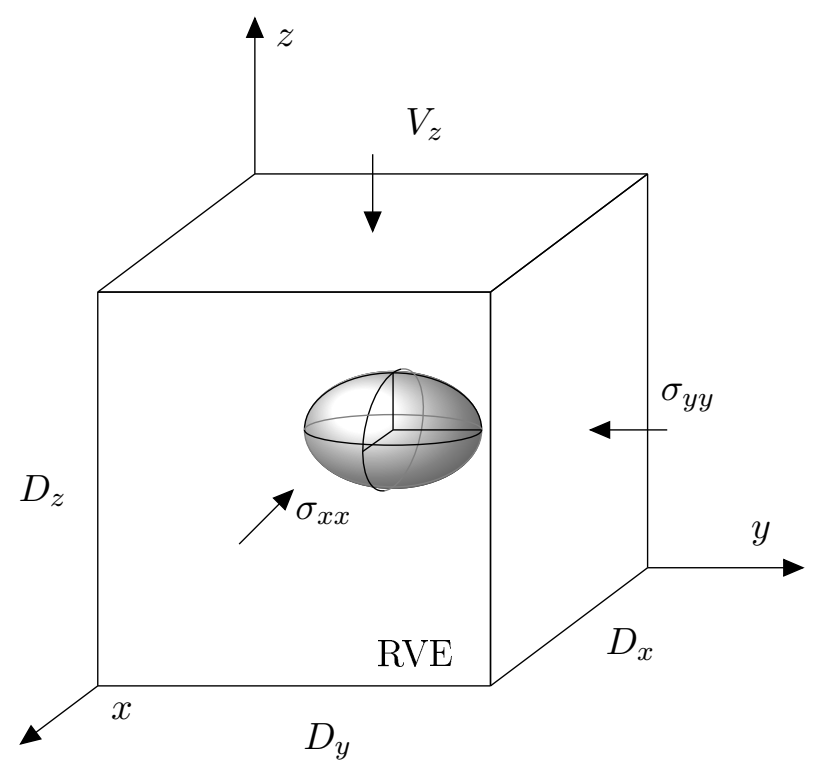

Figure 3: Representative Volume Element containing an ellipsoidal void, and prescribed boundary conditions.

axisymmetric conditions $\sigma_{x x}=\sigma_{y y}$. The matrix material is isotropic and the void volume fraction is very low. By neglecting the effect that might have the void on the global deformation of the RVE, it comes $\varepsilon_{x x} \approx \varepsilon_{y y}$. Equivalent strain therefore reduces to:

$$
\bar{\varepsilon} \approx\left|\varepsilon_{z z}\right|=\left|\ln \left(\frac{D_{z}^{0}-D_{z}}{D_{z}^{0}}\right)\right|,
$$

where $D_{z}$ and $D_{z}^{0}$ are the current height and the initial height of the RVE, respectively. From the definition of stress triaxiality ratio, the condition $\sigma_{x x}=\sigma_{y y}$ leads to:

$$
T_{X}=\frac{\sigma_{m}}{\sigma_{e}}=\frac{\sigma_{x x}+\sigma_{y y}+\sigma_{z z}}{3 \sigma_{e}}=\frac{2 \sigma_{x x}+\sigma_{z z}}{3 \sigma_{e}},
$$


and equivalent von Mises stress reduces to:

$$
\sigma_{e}=\frac{1}{\sqrt{2}} \sqrt{\left(\sigma_{x x}-\sigma_{y y}\right)^{2}+\left(\sigma_{y y}-\sigma_{z z}\right)^{2}+\left(\sigma_{x x}-\sigma_{z z}\right)^{2}}=\left|\sigma_{x x}-\sigma_{z z}\right| .
$$

It comes:

$$
\begin{gathered}
\sigma_{x x}=\left(T_{X}+\frac{1}{3}\right) \sigma_{e} \quad \forall \sigma_{z z} \leq \sigma_{x x}, \\
\sigma_{x x}=\left(T_{X}-\frac{1}{3}\right) \sigma_{e} \quad \forall \sigma_{z z} \geq \sigma_{x x} .
\end{gathered}
$$

In Eq. 5, the values of $\sigma_{x x}$ (and thus the ones of $\sigma_{y y}$ ) to be prescribed are calculated using the current global equivalent von Mises stress in the RVE. The latter is given by the material behaviour in Eq. 1, according to the global strain condition in Eq. 2 that is directly prescribed by the boundary condition $V_{z}$.

\section{Parametric sensitivity study}

Equivalent strain and stress triaxiality states are considered in most models from literature. Material parameters are considered in the analytical and semi-empirical models. However, there is at present a lack of models being able to consider geometrical aspects of the voids, although it was qualitatively shown as a key parameter (Lee and Mear, 1994; Saby et al., 2013). Geometry is thus mainly addressed within this work.

A wide campaign of FE simulations at the RVE scale was defined and is presented in this section. The main objective is thus to clearly identify the parameters with first-order influence on void closure.

First, thirteen ellipsoids were defined using different orientations and the effect of spatial orientation was observed. Then, seven various ellipsoidal geometries were defined using various aspect ratios in order to study the effect of geometry for three key orientations. The effect of mechanical state was then studied for various geometries. For each geometrical case, eight different stress triaxiality ratios were successively applied on the RVE over the range $T_{X}=[-1,0]$. This range was previously identified as representative of most hot forging and hot rolling processes, based on a preliminary study of process simulations (Saby, 2013). The influence of material parameters was also studied in terms of strain hardening/softening and strain-rate sensitivity.

The Hansel-Spittel law (Eq. 1) was used with constants that are typical for the behaviour of hot steels. The stress-strain curve obtained in the given mechanical conditions is illustrated in Fig. 4.

\subsection{Sensitivity to void orientation}

Let us define the canonical basis $\left(\overrightarrow{e_{x}}, \overrightarrow{e_{y}}, \overrightarrow{e_{z}}\right)$, see Fig. 1 . The three principal vectors of deformation $\left(\overrightarrow{e_{1}}, \overrightarrow{e_{2}}, \overrightarrow{e_{3}}\right)$ can be obtained by diagonalizing the strain-rate tensor. According to the boundary conditions that are applied on the RVE (see Fig. 3), the main compression direction systematically coincides with the $z$-axis. The three principal vectors for the void geometry $\left(\overrightarrow{u_{1}}, \overrightarrow{u_{2}}, \overrightarrow{u_{3}}\right)$ in Fig. 1 are defined as the eigenvectors of the void's inertia matrix.

The orientation of a void is defined with respect to the main compression direction $\overrightarrow{e_{1}}$, using parameters $\left(p_{1}, p_{2}, p_{3}\right)$ such as:

$$
\begin{aligned}
& p_{1}=\left(\overrightarrow{u_{1}} \cdot \overrightarrow{e_{1}}\right)^{2}, \\
& p_{2}=\left(\overrightarrow{u_{2}} \cdot \overrightarrow{e_{1}}\right)^{2}, \\
& p_{3}=\left(\overrightarrow{u_{3}} \cdot \overrightarrow{e_{1}}\right)^{2},
\end{aligned}
$$




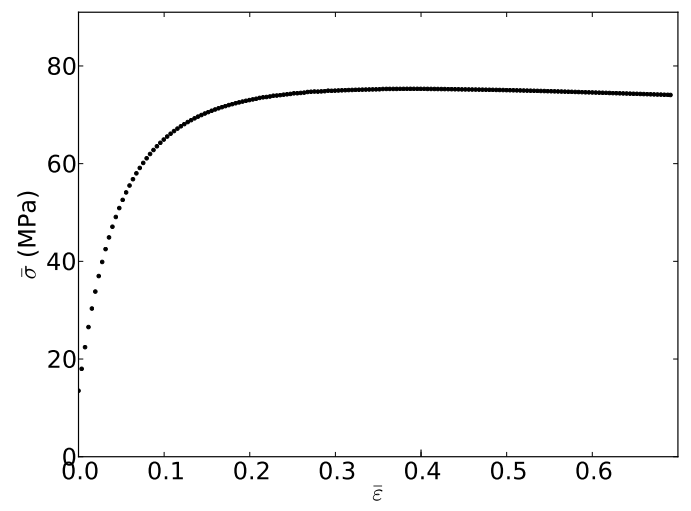

Figure 4: Stress-strain curve of the the material used for the calibration (Eq. 1).

This definition verifies $p_{1}+p_{2}+p_{3}=1$.

In Fig. 1, the particular configuration $(1,0,0)$ is illustrated, meaning that the principal direction $\overrightarrow{u_{1}}$ of the ellipsoid is colinear with the principal deformation direction $\overrightarrow{e_{1}}$.

Several orientations were defined in order to study the influence of orientation on void closure. A given ellipsoid of dimensions $r_{2} / r_{1}=2$ and $r_{3} / r_{1}=1.5$ was used. The cases presented in Fig. 5 and 6 were obtained by rotating the void around the $\overrightarrow{e_{y}}$ axis and the $\overrightarrow{e_{x}}$ axis, respectively.

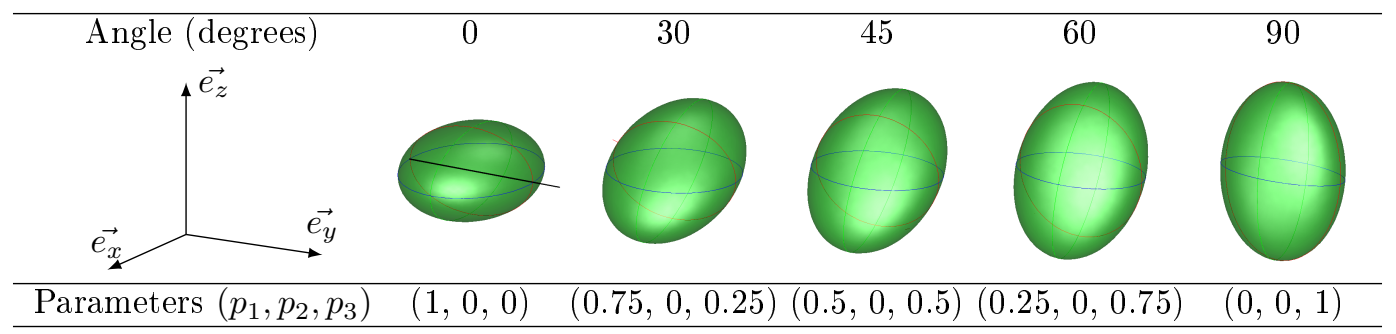

Figure 5: Various ellipsoids, obtained by rotation around $\overrightarrow{e_{y}}$.

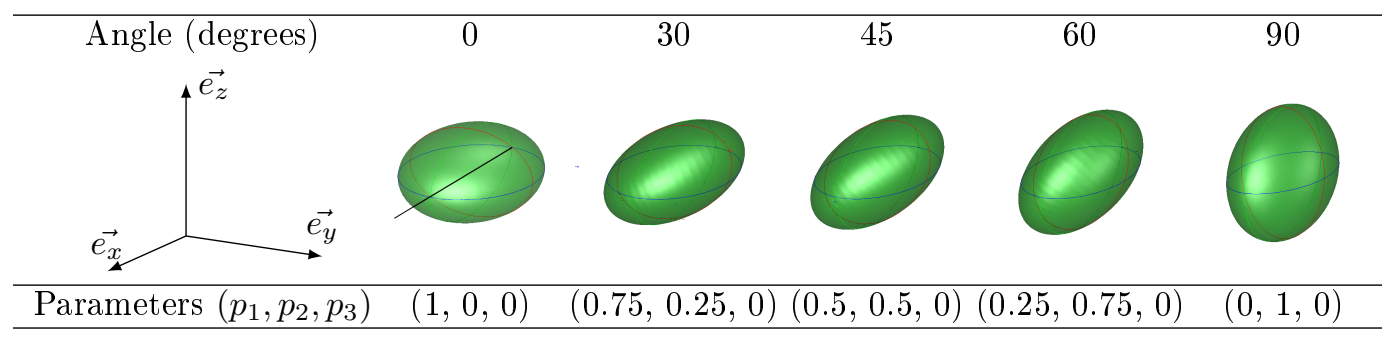

Figure 6: Various ellipsoids, obtained by rotation around $\overrightarrow{e_{x}}$.

The void volume evolutions for each series of cases are presented in Fig. 7. Void volume is expressed as $V / V_{0}$, where $V$ is the current measured volume in the RVE and $V_{0}$ its initial value. The orientation is given using the couple of values $\left(p_{1}, p_{2}\right)$, since $p_{3}$ can be deduced from $p_{3}=1-p_{1}-p_{2}$.

From the curves, it can be seen that orientation has a significant influence on void volume evolution. Three particular cases can be pointed out: the case $\left(p_{1}, p_{2}\right)=(1,0)$, the case $\left(p_{1}, p_{2}\right)=$ $(0,1)$ and the case $\left(p_{1}, p_{2}\right)=(0,0)$. Each case corresponds to a compression along $\overrightarrow{u_{1}}, \overrightarrow{u_{2}}$ and $\overrightarrow{u_{3}}$, 
respectively. The curves indicate that compression along $\overrightarrow{u_{1}}$ involves the fastest void closure, as the void is compressed along its smallest dimension $r_{1}$. On the contrary, compression along $\overrightarrow{u_{3}}$ involves the slowest void closure, as compression is made along the void's longest dimension $r_{3}$. These two cases can be seen as upper and lower bounds for these given ellipsoid dimensions. Void evolution under compression along $\overrightarrow{u_{2}}$, as well as for any other orientation, are naturally between these bounds.

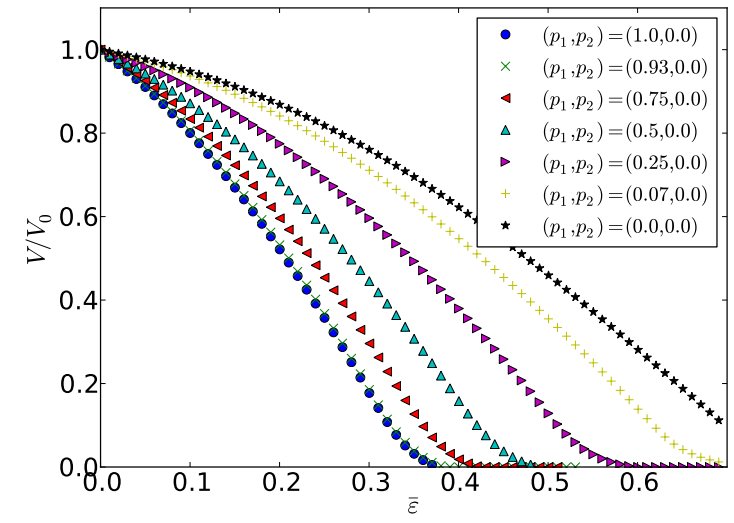

(a) Rotation around $\overrightarrow{e_{y}}\left(p_{2}=0\right)$

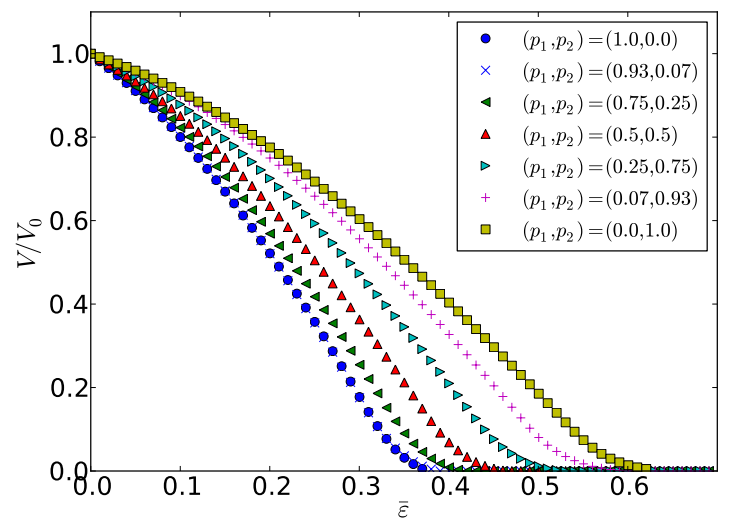

(b) Rotation around $\overrightarrow{e_{x}}\left(p_{3}=0\right)$

Figure 7: Influence of orientation parameters.

\subsection{Sensitivity to void's aspect ratios}

The upper and lower bounds obtained previously using a given ellipsoid evidently depends on the initial geometry. In order to assess the influence of geometry on these bounds, several ellipsoids were defined according to various aspect ratios. The aspect ratios are defined by $\left(\frac{r_{3}}{r_{1}}, \frac{r_{2}}{r_{1}}\right)$.

Various aspect ratios were used and are presented in Fig. 8 (using the orientation $\left(p_{1}, p_{2}\right)=$ $(1,0))$. Ellipsoids A and B are prolate, since $r_{1}=r_{2}<r_{3}$. Ellipsoids $\mathrm{C}$ and D are oblate, since $r_{1}<r_{2}=r_{3}$. Ellipsoids $\mathrm{E}$ and $\mathrm{F}$ are defined such as $r_{1}<r_{2}<r_{3}$. For each ellipsoid, the RVE was generated and identical deformation conditions were applied in order to obtain void volume evolutions along the three principal directions of each ellipsoid.

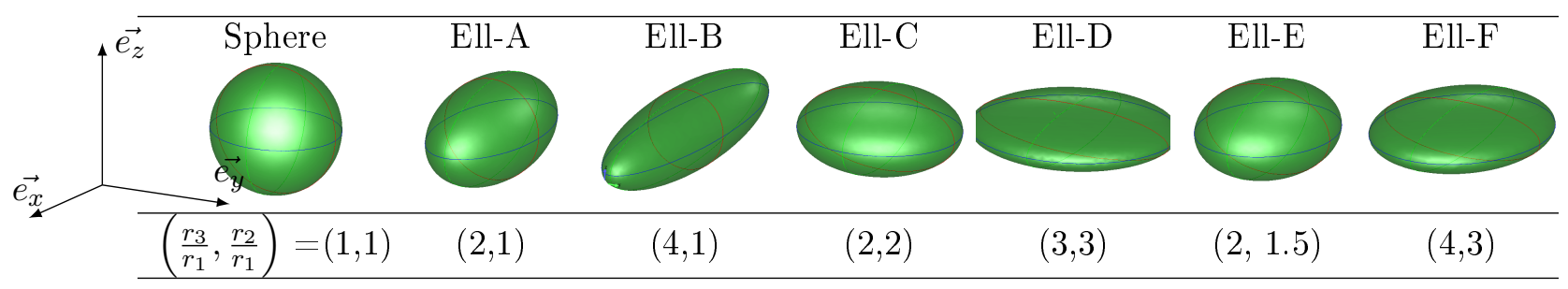

Figure 8: Various ellipsoids with given aspect ratio $\left(\frac{r_{3}}{r_{1}}, \frac{r_{2}}{r_{1}}\right)$, for $\left(p_{1}, p_{2}\right)=(1,0)$.

The void volume evolutions are given in Fig. 9. In the case of ellipsoids A, B, C and D, two of the three curves (corresponding to the three orientations) are superimposed. This is due to the particular symmetry of such geometries. For the prolate ellipsoid A, identical behaviours are 
obtained for compressions along the $\overrightarrow{u_{1}}$ and $\overrightarrow{u_{2}}$ axes. This result is observed for the prolate ellipsoid $\mathrm{B}$ as well. For the oblate ellipsoids $\mathrm{C}$, identical behaviours are obtained for compressions along the $\overrightarrow{u_{2}}$ and $\overrightarrow{u_{3}}$ axes. The same behaviour is observed for the oblate ellipsoid D. For ellipsoids E and F, the closure rates along one direction depends on the geometry.

By comparing the pairs of ellipsoids (A, B), (C, D) and (E, F), it is observed that larger aspects ratios tend to increase the difference in terms of closure rate between the minor axis and the major axis.

By comparing the triplet of ellipsoids (A, C, E) in the orientation $(1,0)$, different closure rates are observed, although their first aspect ratio are identical $\frac{r_{3}}{r_{1}}=2$. This remark shows that void closure not only depends on one aspect ratio but on the tridimensional morphology.

\subsection{Sensitivity to stress triaxiality ratio}

As described in section 2.2, boundary conditions are applied in order to prescribe a constant stress triaxiality ratio. Void closure was studied under various stress triaxiality ratios and the curves are presented in Fig. 10.

The curves confirm that compressive stress states have a favorable effect on void closure. This result is in good agreement with existing models (STB models, analytical models and semi-empirical models).

\subsection{Final stages of closure}

When looking at the curves in Fig. 7, 9 and 10, it can be observed that void volume generally exhibits a slight change of slope at final stages. This phenomenon is due to the presence of contact between internal void surfaces at the final stage of closure, as shown in Fig. 11. This phenomenon was also observed by Zhang et al. (2009) for spherical voids.

The change of slope also depends on the void's morphology. It remains moderate for spherical and ellipsoidal voids but might increase in the case of very tortuous voids (Saby et al., 2013). Nevertheless, for most industrial cases, although the use of equivalent ellipsoids remains unsufficient to faithfully represent the final behaviour of real void, very satisfactory results were obtained regarding the void volume evolution over a range $V / V_{0} \in\left[V_{\text {crit }}, 1\right]$. The value $V_{\text {crit }}$ may vary between 0.4 and 0.2 depending on the tortuosity of the considered void.

An accurate quantification of the tortuosity would therefore be required in order to address its effect on final stages of void closure. In the present work, the sensitivity study to geometrical parameters is based on FE simulations of RVE containing ellipsoidal voids, and the range of interest is defined as $V / V_{0}=[0.2,1]$. The final stages were thus ignored in this work for the construction of the model.

\subsection{Sensitivity to material parameters}

A dependence to material behaviour was pointed out in the literature. For visco-plastic materials, Duva and Hutchinson (1984) studied the effect of strain-rate sensitivity coefficient using various stress triaxiality conditions for the evolution of a spherical void. They used a power-law matrix without strain hardening $\sigma_{0}=A \dot{\bar{\varepsilon}}^{m}$ and proposed an analytical dependence of void evolution to strain-rate sensitivity coefficient $m$. The authors stated that the effect of strain-rate sensitivity for such materials can also be applied for the case of strain hardening materials obeying $\sigma_{0}=A \bar{\varepsilon}^{n}$. In fact, the involved mechanisms are the same and the same analysis can be conducted by simply replacing $\dot{\bar{\varepsilon}}$ by $\bar{\varepsilon}$. 


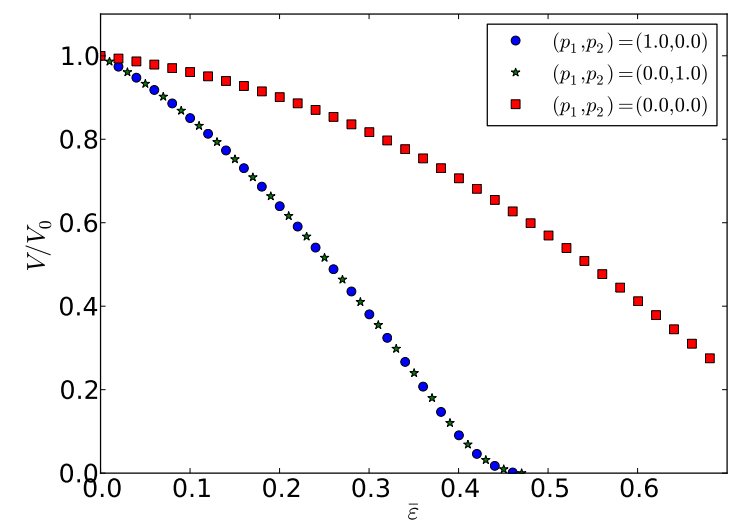

(a) Ellipsoid A

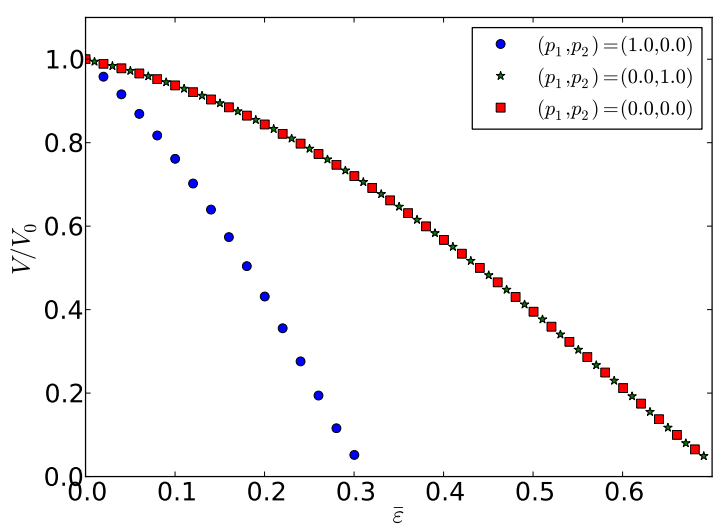

(c) Ellipsoid C

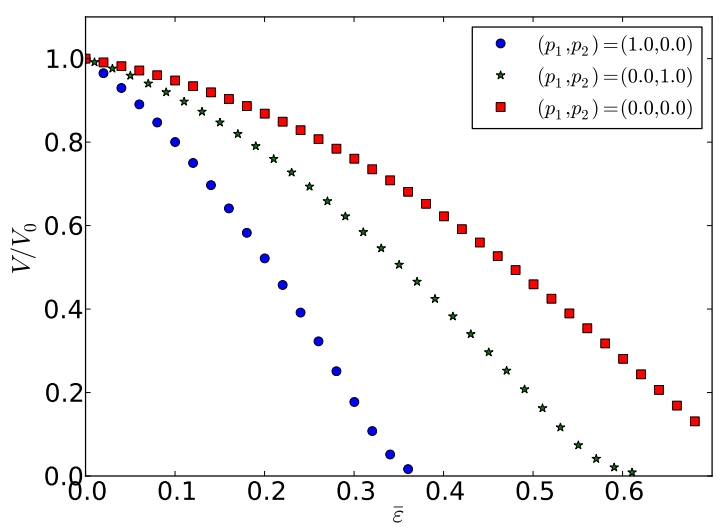

(e) Ellipsoid E

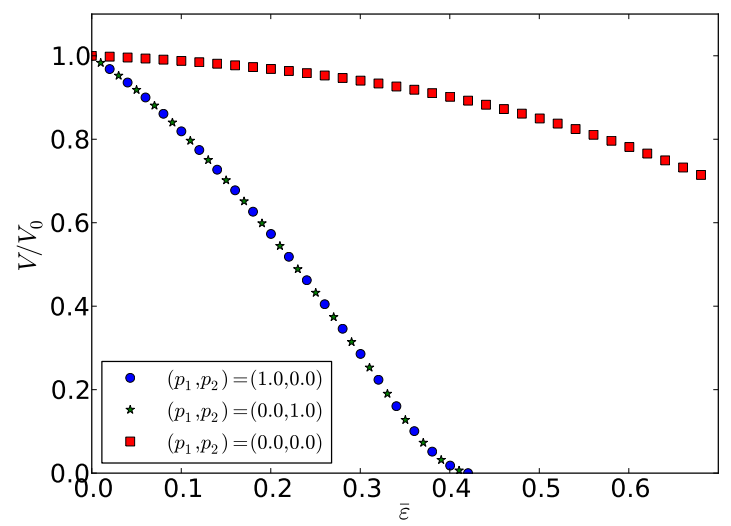

(b) Ellipsoid B

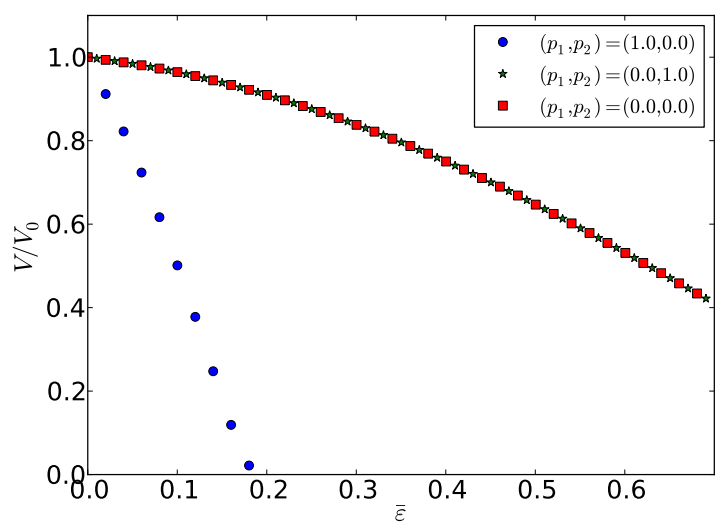

(d) Ellipsoid D

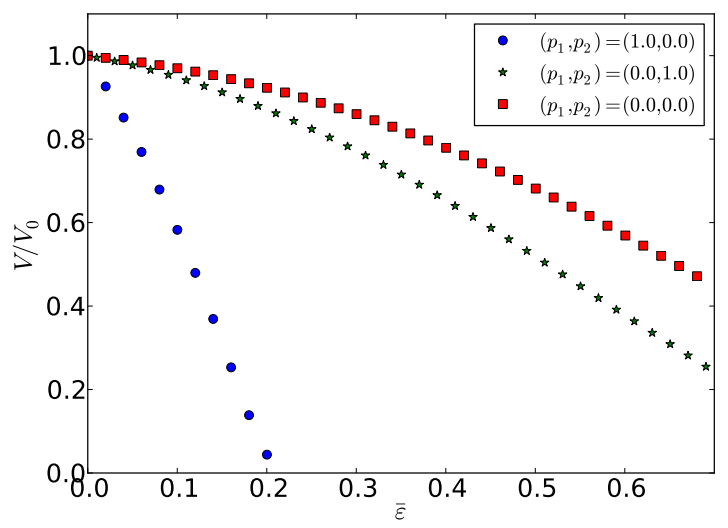

(f) Ellipsoid F

Figure 9: Void volume evolution for various initial geometries, and for three different orientations.

Ragab (2004) studied the effect of strain hardening on void opening, dealing with damage prediction. The author has shown that strain hardening mainly affects the coalescence phenomenon. 


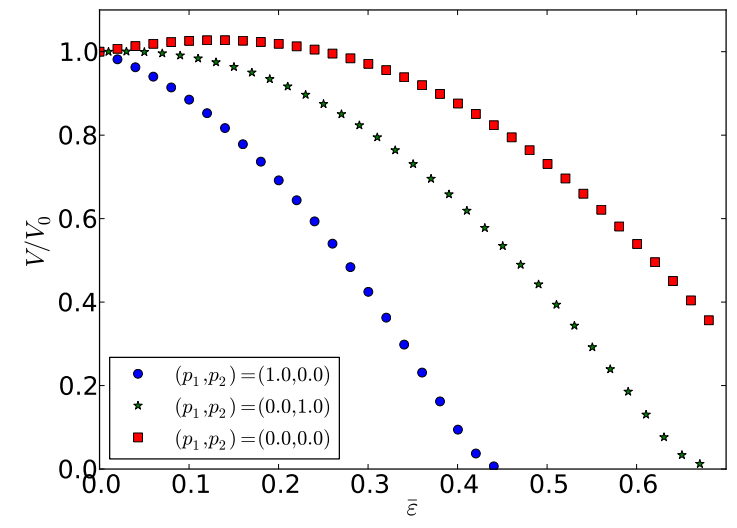

(a) $T_{X}=0$

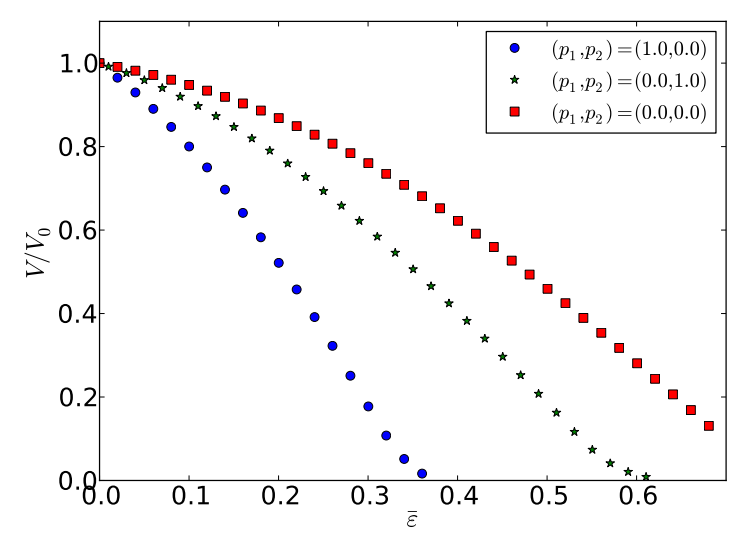

(c) $T_{X}=-1 / 3$

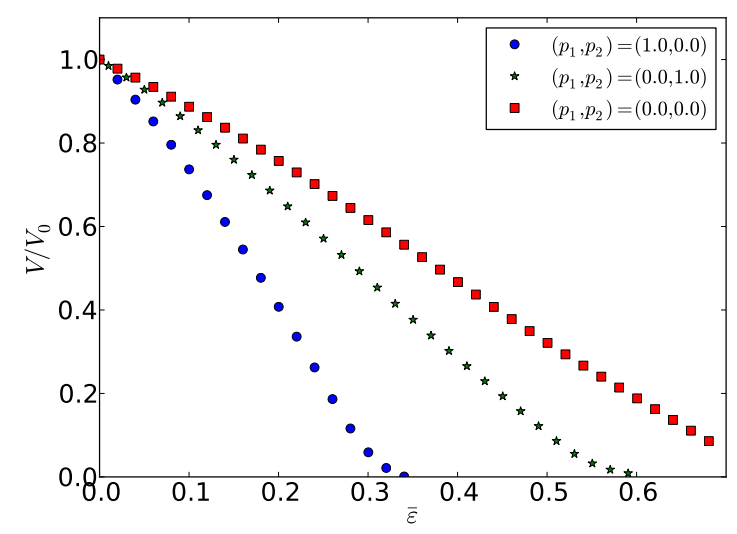

(e) $T_{X}=-0.6$

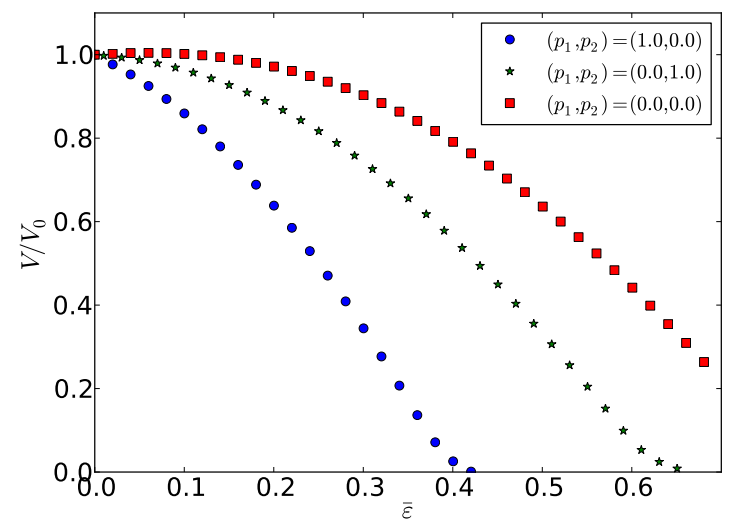

(b) $T_{X}=-0.1$

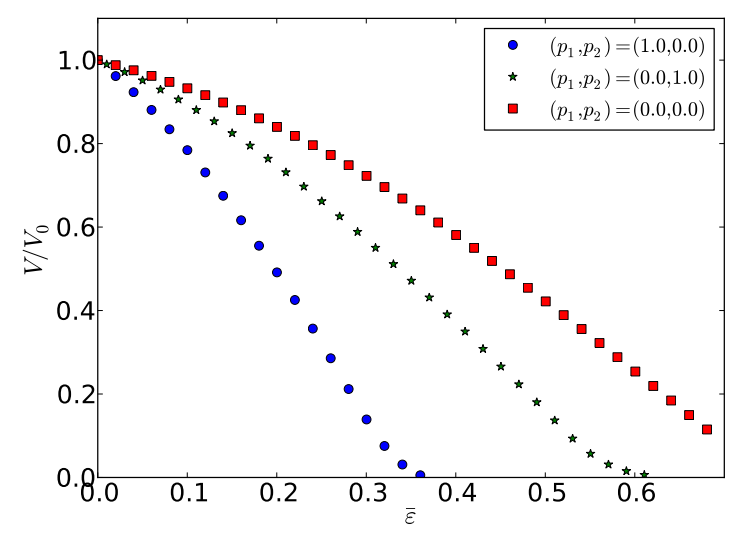

(d) $T_{X}=-0.4$

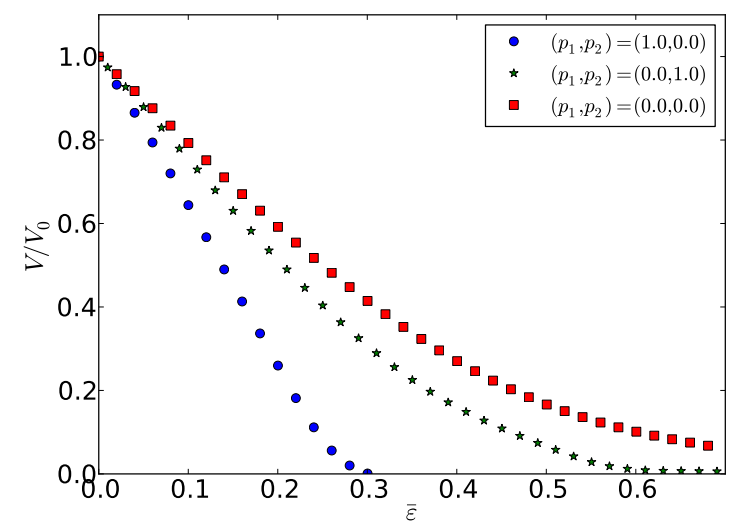

(f) $T_{X}=-1.0$

Figure 10: Void volume evolutions of Ell-E, under various values of stress triaxiality ratios. 

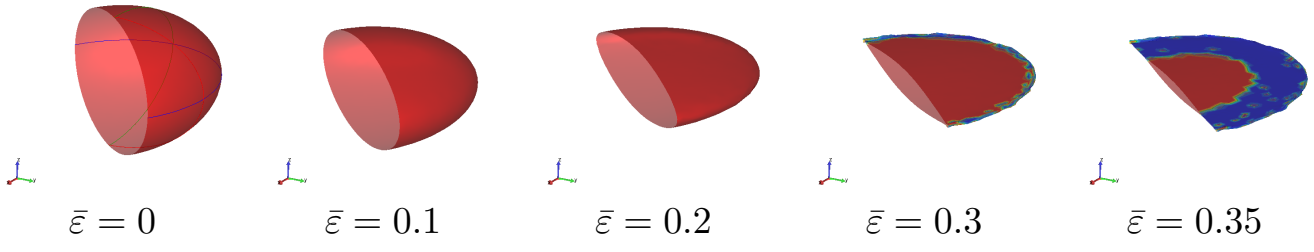

Figure 11: Evolution of the void Ell-E during closure for the orientation $\left(p_{1}, p_{2}\right)=(1,0)$ under $T_{X}=-1 / 3$. The blue color shows the contact between internal surfaces.

A slight effect was observed on void evolution (in this case, growth) as well. This results is in good agreement with the remark from Duva and Hutchinson (1984).

In this section, the effect of material parameters from Eq. 1 are studied on the closure behaviour of a spherical void.

\subsection{Influence of material consistency}

Using an arbitrary strain hardening coefficient $(n=1)$, material laws with different values of $A=\{1,2,10,100\} \mathrm{MPa}$ were compared. The stress-strain curves are plotted in Fig. 12a. The resulting void volume evolutions are plotted in Fig. 12b. All closure behaviours are identical in all cases, regardless of the value of $A$.

A second case was defined using another value of strain hardening coefficient $n=0.1$, and for the values $A=\{1,10\} \mathrm{MPa}$. The stress-strain curves and the resulting void volume evolution are plotted in Fig. 12c and Fig. 12d, respectively. As both void volume evolutions are again superimposed, the same conclusion can be made. The material consistency $A$ has no influence on void closure. An arbitrary value $A=1 \mathrm{MPa}$ is thus set for the following analyses.

\subsubsection{Effect of strain-rate sensitivity $m$}

In order to focus on the pure effect of $m$, the Hansel-Spittel law can be used with the parameters $n=m_{4}=\varepsilon_{0}=0$ (see Eq. 1). A rigid perfectly plastic material is obtained, such as the one used in the analytical solutions of Duva and Hutchinson (1984) and the semi-empirical model of Zhang et al. (2009).

Within this work, due to convergence difficulties when using rigid perfectly plastic behaviour $(n=0)$ in FORGE (2011), the value $n=0.01$ was used instead. Several values of $m$ were defined on the range $[0.01,1]$ and the resulting void volume evolutions are plotted in Fig. 13.

The curves show a significant influence of $m$ on void closure behaviour. The results are in rather good agreement with the values obtained from the model of Zhang et al. (2009).

Although the effect is significant on the whole tested range $m=[0.01,1]$, the values of $m$ for hot metals rarely exceed $m=0.25$. Over the range $m=[0.01,0.2]$, the effect of $m$ on void volume evolution remains moderate.

\subsubsection{Effect of strain hardening/softening $n$}

In order to study the pure effect of strain hardening $n$, the Hansel-Spittel equation is used with the parameters $m=m_{4}=\varepsilon_{0}=0$. Several values of $n$ over the range $[0.01,1]$ were defined to cover a large variety of material behaviours.

Softening behaviour can be introduced in the Hansel-Spittel law using negative values of $n$. Another case with the values $\left(n, m_{4}\right)=(-0.1,-0.001)$ was thus added in order to qualitatively 


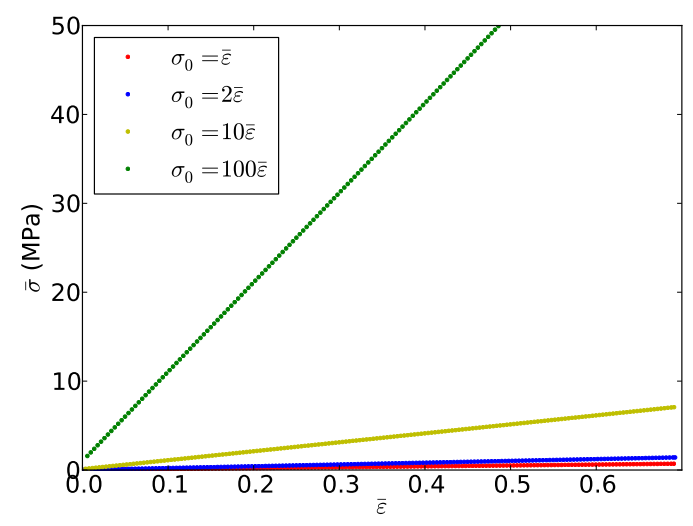

(a) Stress vs. strain curves, for $n=1$

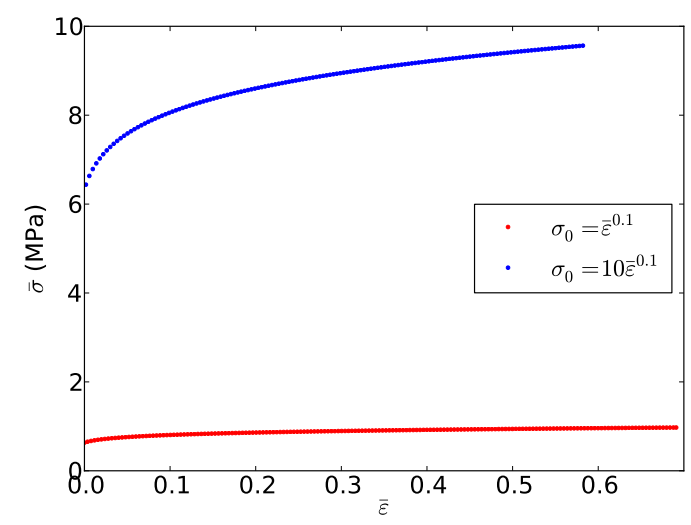

(c) Stress vs. strain curves, for $n=0.1$

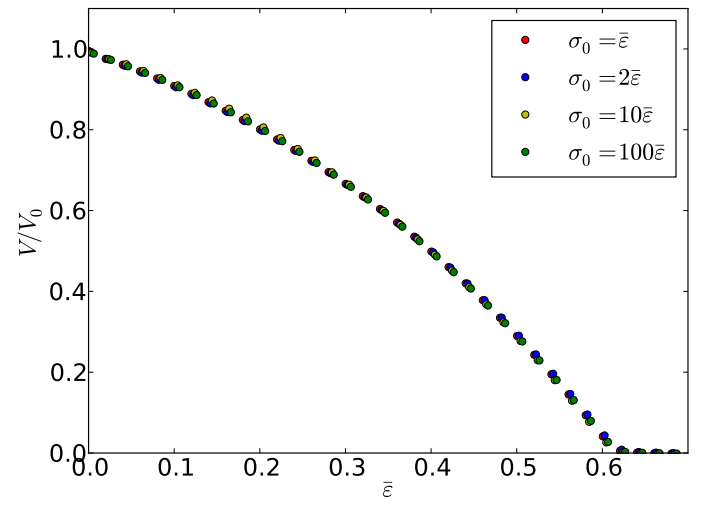

(b) Void volume evolution, for $n=1$

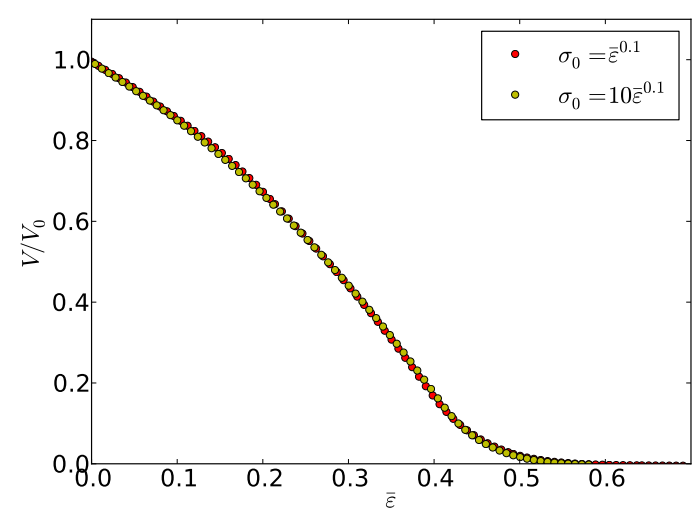

(d) Void volume evolution, for $n=0.1$

Figure 12: Effect of material consistency $A$ on void volume evolution, under $\dot{\bar{\varepsilon}}=1 \mathrm{~s}^{-1}$ and $T_{X}=-1 / 3$, for different values $A=\{1,2,10,100\} \mathrm{MPa}$, using $n=1$ in (a,b) and using $n=0.1$ in (c,d).

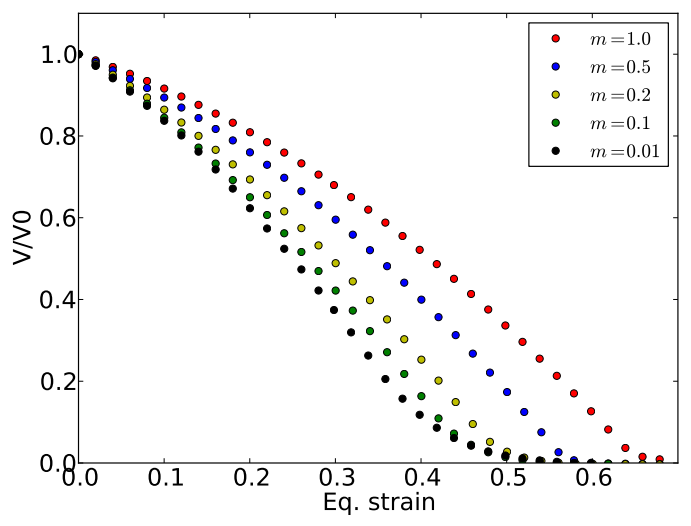

Figure 13: Effect of strain-rate sensitivity $m$ on void volume evolution, using $n=0.01$ under $\dot{\bar{\varepsilon}}=1 \mathrm{~s}^{-1}$ and $T_{X}=-1 / 3$.

represent a material with softening behaviour. Note that in this particular case, a non-zero value of $m_{4}$ must be set in order to avoid the presence of an infinite value of $\sigma_{0}$ at $\bar{\varepsilon}=0$. It was verified that 
the introduction of the non-zero value of $m_{4}$ has no significant impact on the material behaviour. Although this particular case may not physically represent a typical hot metal behaviour, it is a simple qualitative example exhibiting a negative slope of the stress-strain curve that is easily comparable to the cases defined above.

The stress-strain curves are presented in Fig. 14a and illustrate the large variety of material behaviours that is considered. The resulting void volume evolutions are plotted in Fig. 14b.

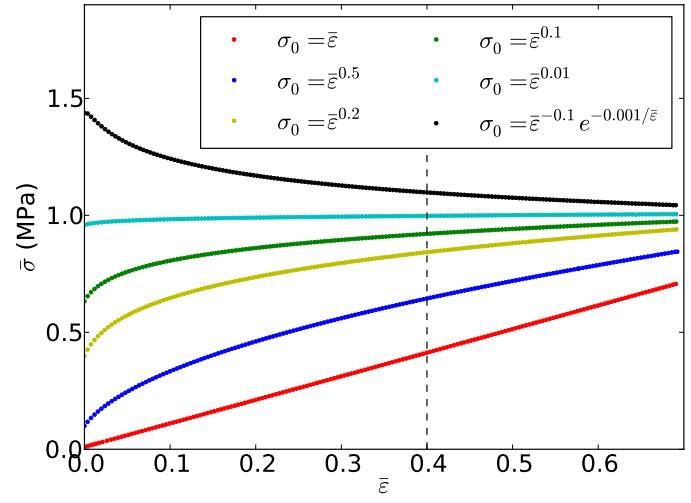

(a) Stress vs. strain curves

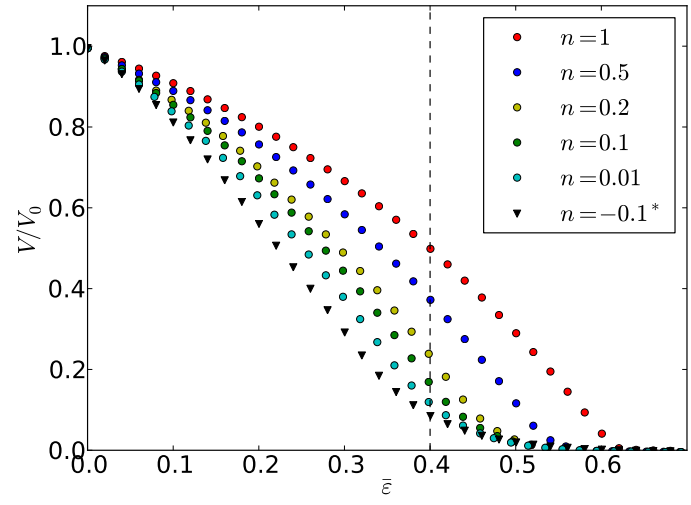

(b) Void volume evolution

Figure 14: Effect of strain hardening on the evolution of a spherical void, using strain-rate sensitivities $m=0$ under $\dot{\bar{\varepsilon}}=1 \mathrm{~s}^{-1}$ and $T_{X}=-1 / 3\left({ }^{*}\right.$ for negative strain hardening values, the value $m_{4}=-10^{-3}$ is used).

The curves in Fig. 14b for various values of $n$ are roughly identical to the ones in Fig. 13 for various values of $m$. As mentioned by Duva and Hutchinson (1984), the effect of strain hardening and the effect of strain-rate sensitivity are driven by the same mechanisms. The resulting influence of $n$ can therefore be used for the one of $m$ and vice-versa.

Fig. 14b also indicates that strain hardening has a significant effect on void volume evolution on the tested range $n=[-0.1,1]$. A difference of about $40 \%$ was measured in terms of void volumes at $\bar{\varepsilon}=0.4$, between the cases $n=-0.1$ and $n=1$. This difference tends to decrease at the final stages, as the slope drops due to internal contact.

The effect of internal contact is illustrated in Fig. 15. In the case of a linear material $n=1$, the void's shape remains ellipsoidal throughout the deformation. When reducing strain hardening $n$, the curvature is changed and contact between internal surfaces appears earlier. This effect induces a faster void closure at the beginning, and, on the contrary, reduces the final slope to complete closure. However, it is noteworthy that the required deformation for complete closure is comparable for all cases.

The values of strain hardening for hot metal rarely exceed the value $n=0.2$. The difference in terms of void volume at $\bar{\varepsilon}=0.4$ reduces to about $15 \%$ over the range $n=[-0.1,0.2]$. In the case of hot metal forming, the effect of strain hardening/softening is thus significantly lower than the effects of void geometry or stress triaxiality ratio.

As a consequence, the effect of strain-rate sensitivity $m$ and of strain hardening/softening $n$ are considered as second-order parameters and will not be addressed in the prediction model presented in this paper. 


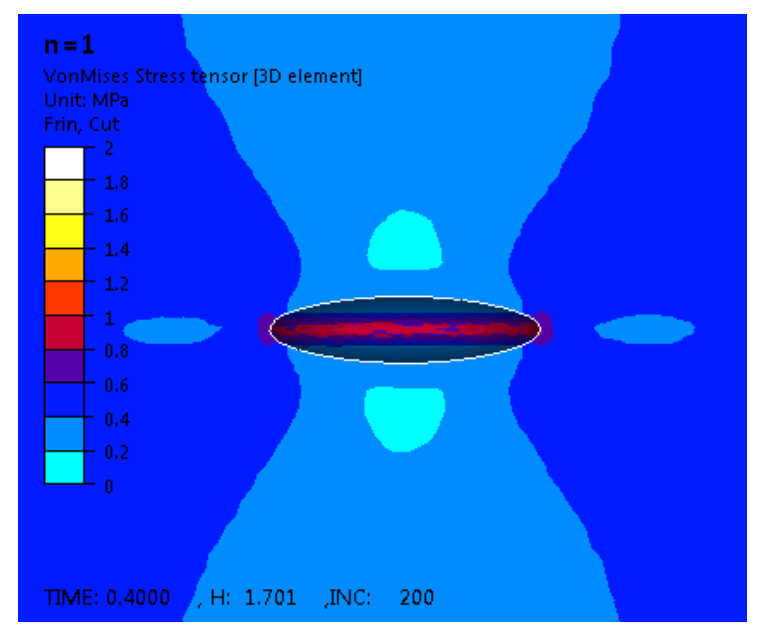

(a) $n=1$

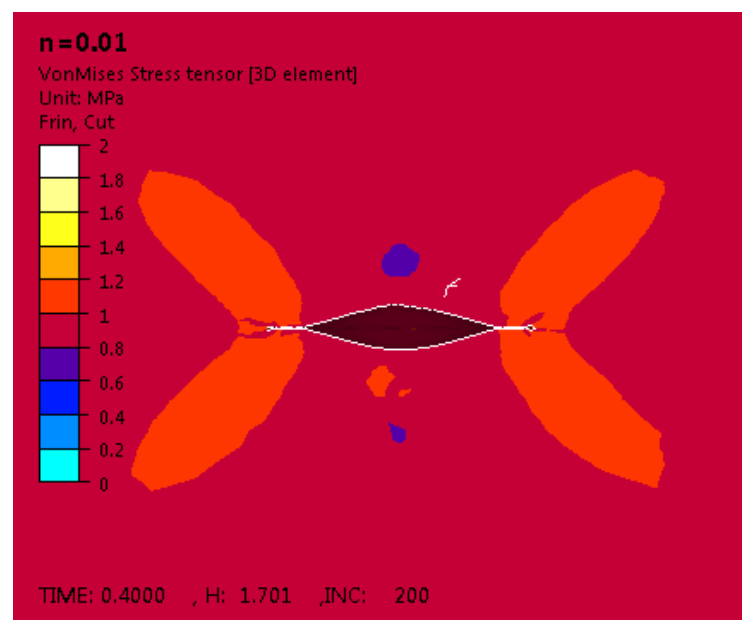

(c) $n=0.01$

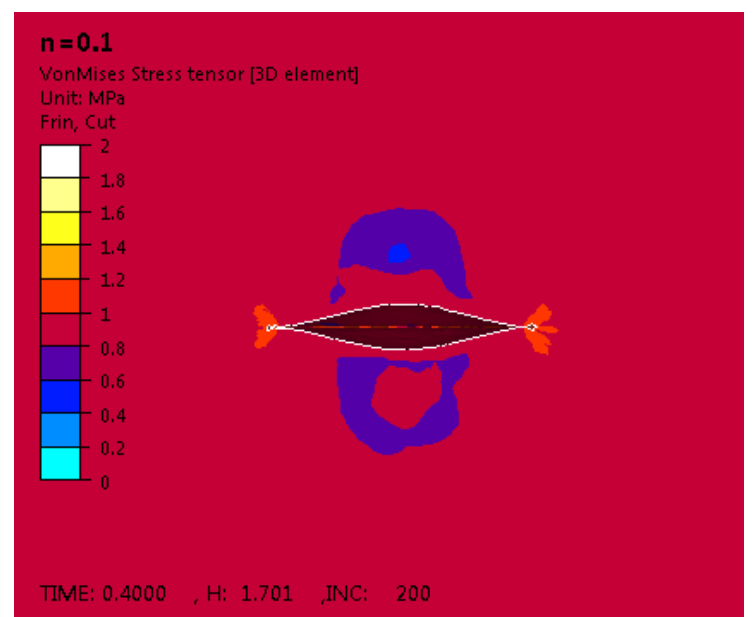

(b) $n=0.1$

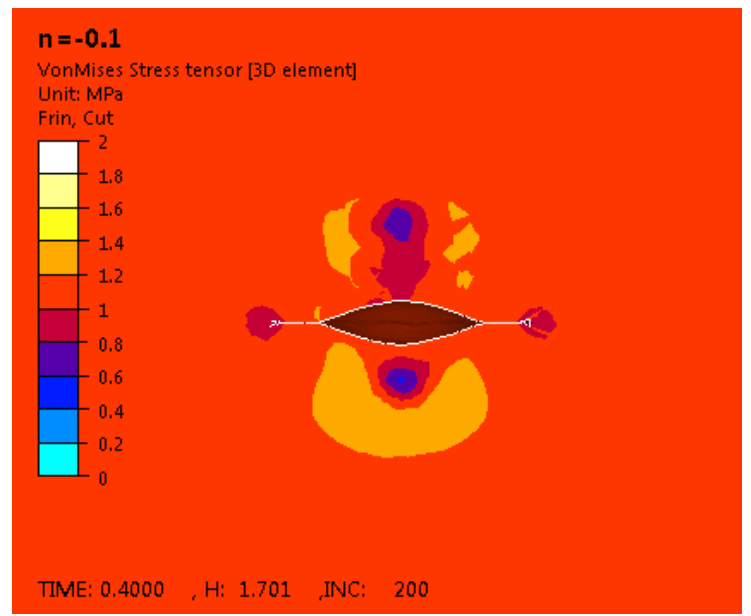

(d) $n=-0.1$

Figure 15: Cutting plane illustrating the von Mises equivalent stress field, for an initially spherical void at $\bar{\varepsilon}=0.4$, under $\dot{\bar{\varepsilon}}=1 \mathrm{~s}^{-1}, T_{X}=-1 / 3$ and for different values of strain hardening coefficient $n$.

\section{Prediction model for void closure}

This section presents the construction steps for the new prediction model for void closure. First, the choice of an analytical function that is able to model void closure as a function of equivalent strain is presented. Then the dependence to void orientation is quantitatively studied in order to extend the analytical function to any orientation of ellipsoid (for a given ellipsoid). The dependence to aspect ratios is then quantitatively studied in order to extend the analytical function to any ellipsoid (geometry and orientation). Finally, the dependence to stress triaxiality ratio is quantitatively studied in order to extend the analytical function to any ellipsoid and to any stress triaxiality ratio. The final equation is verified using four geometry-equivalent ellipsoids (obtained from real observations).

The analytical functions are calibrated using the RVE simulations from the campaign presented in the previous section, using regression analysis. The regressions accuracy is systematically il- 
lustrated using the $R^{2}$-correlation coefficient. The final difference that is obtained between the calibrated prediction model and the reference states (from RVE simulations) is given using the $L^{2}$-norm.

\subsection{Choice of an analytical function}

A relationship between void volume and equivalent strain is sought here. According to the parabolic aspects of the curves obtained in the previous section for various geometries and under various conditions, a second order polynomial function is proposed. The analytical function can be expressed as:

$$
V / V_{0}=A+B \bar{\varepsilon}+C \bar{\varepsilon}^{2} .
$$

where $A, B$ and $C$ are fitting parameters. The initial condition leads to $A=1$. This expression can be reduced to a linear expression:

$$
\frac{V / V_{0}-1}{\bar{\varepsilon}}=B+C \bar{\varepsilon},
$$

in which the two coefficients $B$ and $C$ can be fitted using linear regression analysis. This is of great interest regarding the large quantity of parameters to be assessed within this study.

As pointed out in the previous section, the final void evolution may not be representative of void closure in the general case, as it is driven by the particular ellipsoidal shape. As a consequence, interest is focused on the range $V / V_{0}=[0.2,1]$. Values below the threshold value 0.2 will therefore be ignored in the regression analysis (see example in Fig. 16). The beginning of the curves is ignored as well due to the presence of a vertical asymptote at $\bar{\varepsilon}=0$ on the linearized curves, and in order to accurately capture the linear evolution using linear regression analysis.

\subsection{Dependence to orientation}

For all orientation cases, the void volume evolutions were fitted using linear regression analysis. It is illustrated for several particular orientations in Fig. 16. Values of $B$ and $C$ coefficients were obtained with very good correlation (using $R^{2}$-correlation coefficients). The values for $B$ and $C$ are plotted with dots in Fig. 17 versus orientation parameters $p_{1}$ and $p_{2}$. Each coefficient exhibits a linear dependence to orientation parameters. Linear regression is thus used to fit this dependence and $R^{2}$-correlation showed very good values as well. As a consequence, the following expression is proposed:

$$
\begin{aligned}
& B=p_{1} B^{\overrightarrow{u_{1}}}+p_{2} B^{\overrightarrow{u_{2}}}+p_{3} B^{\overrightarrow{u_{3}}} \\
& C=p_{1} C^{\overrightarrow{u_{1}}}+p_{2} C^{\overrightarrow{u_{2}}}+p_{3} C^{\overrightarrow{u_{3}}} .
\end{aligned}
$$

In this equation, $B^{\overrightarrow{u_{i}}}$ and $C^{\overrightarrow{u_{i}}}$ are geometry-dependent parameters that correspond to void evolution under compression along $\overrightarrow{u_{i}}$. They can be obtained using the three particular cases $\left(p_{1}, p_{2}\right)=(1,0)$, $\left(p_{1}, p_{2}\right)=(0,1)$ and $\left(p_{1}, p_{2}\right)=(0,0)$, respectively. According to the analytical expression in Eq. 9, for a given ellipsoid, knowing its volume evolution along its three principal directions is sufficient for predicting its volume evolution in any random orientation. The values of $B^{\overrightarrow{u_{i}}}$ and $C^{\overrightarrow{u_{i}}}$ are thus obtained from the regression shown in Fig. 16.

Based on $B^{\overrightarrow{u_{i}}}$ and $C^{\overrightarrow{u_{i}}}$ coefficients that were obtained with Ell-E for $\left(p_{1}, p_{2}\right)=\{(1,0) ;(0,1)$; $(0,0)\}$, the validity of Eq. 9 was checked using randomly oriented ellipsoids. Four orientations were defined using successive rotations around $\overrightarrow{e_{x}}$ and $\overrightarrow{e_{y}}$. The resulting pairs of orientation parameters $\left(p_{1}, p_{2}\right)$ are $(0.56,0.19),(0.19,0.56),(0.19,0.06)$ and $(0.06,0.19)$. The model was computed using 

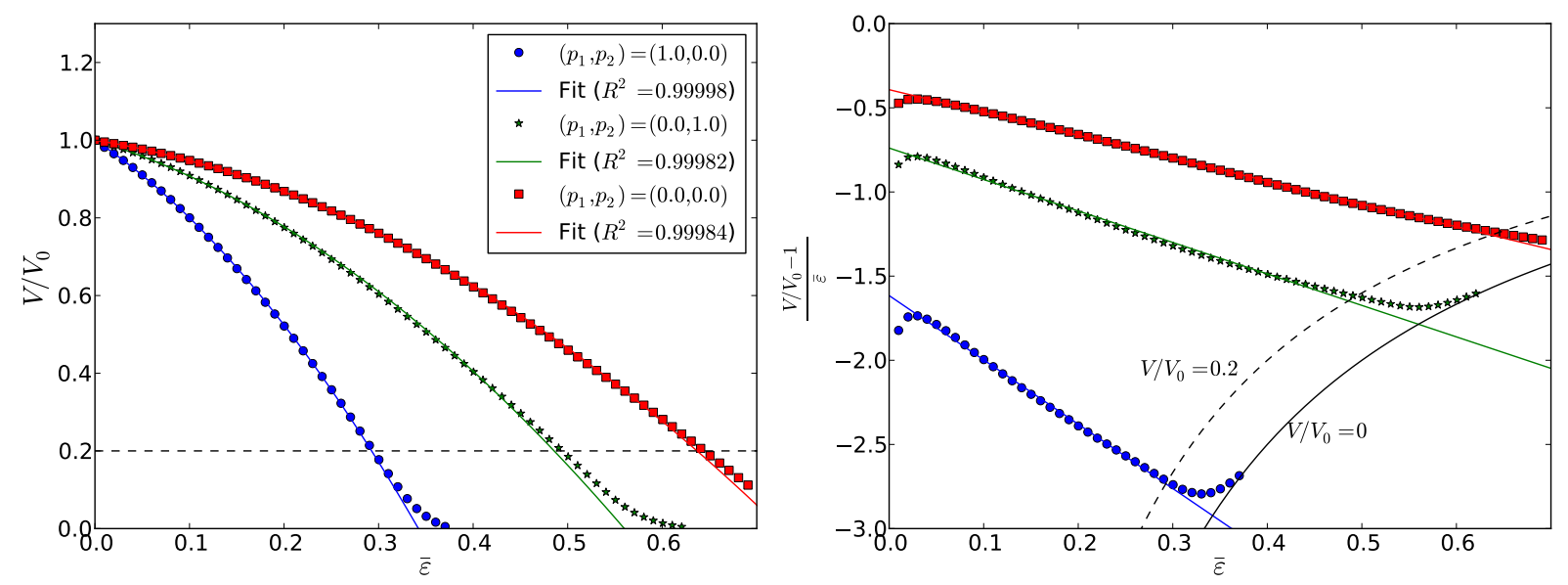

Figure 16: Curve fitting (with $R^{2}$-correlation factor) performed on RVE results (Ell-E for the three principal orientations) by linear regression using the linearized expression (Eq. 8).

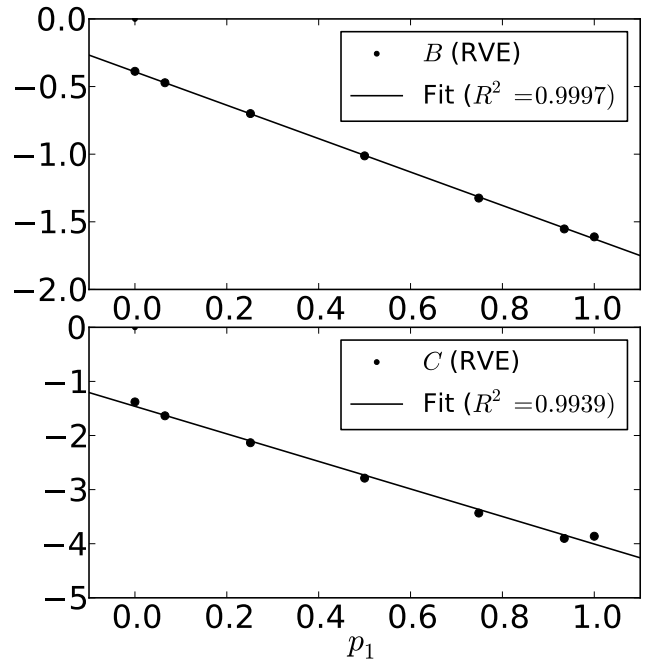

(a) $p_{2}=0$

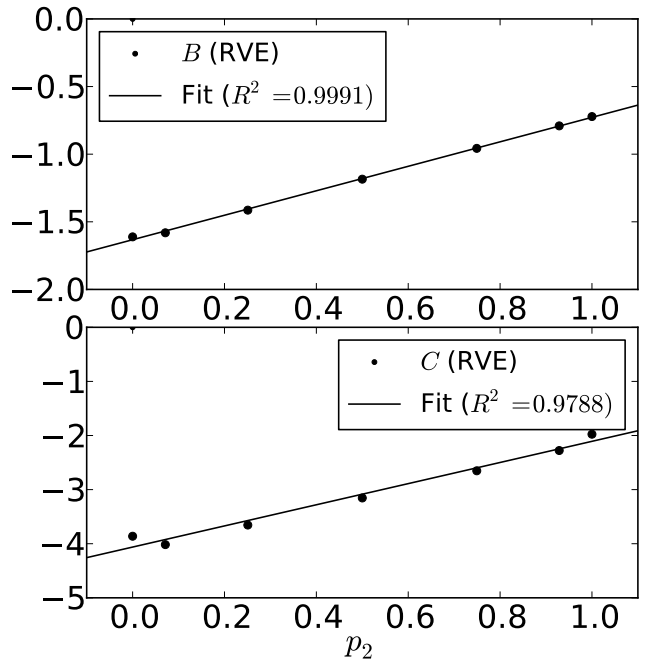

(b) $p_{3}=0$

Figure 17: Influence of orientation parameters on $B$ and $C$ coefficients.

Eqs. 7 and 9 for each orientation. A comparison is given in Fig. 18, together with bound cases $(1,0)$ and $(0,0)$. For the six curves, a very good agreement was obtained between the model and the values obtained from RVE simulations. The average $L^{2}$-norm error was computed over the whole deformation, between the RVE-values and the model in Eq. 9, and remains under 3\% for all cases. As a consequence, the model is suitable to take into account the effect of orientation. Together with Eq. 7 the model is suitable for predicting void evolution for any orientation.

\subsection{Dependence to geometry}

The dependence to aspect ratios is now studied using the same methodology as described above. Void evolution coefficients $B$ and $C$ are obtained from curves in Fig. 9 using linear regression 


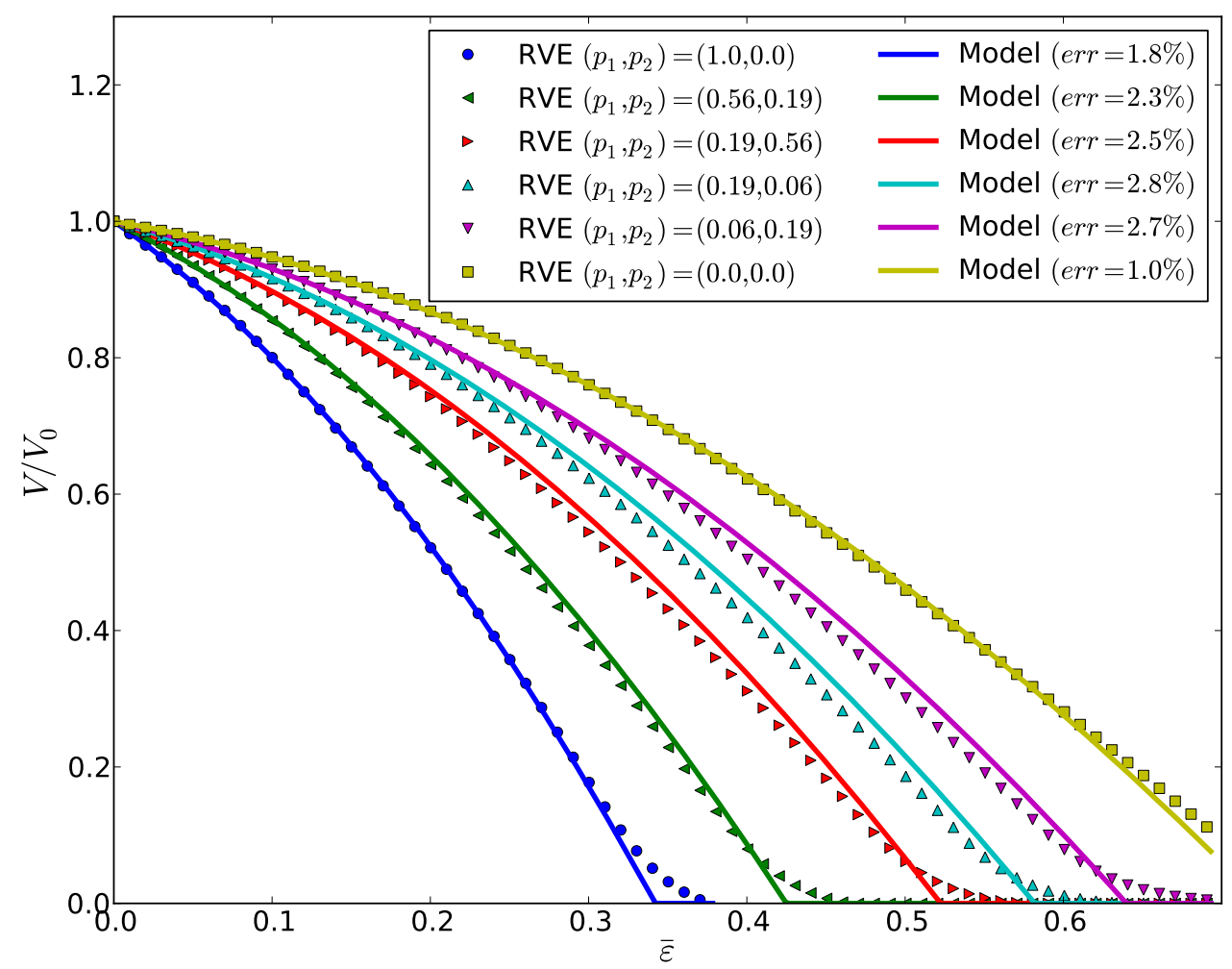

Figure 18: Void volume evolution obtained using RVE simulations (dots) and model predictions (lines) given by Eqs. 7 and 9, for various orientations, with average $L^{2}$-norm error.

analysis. The linearized form of void volume evolution is used as well, such as shown in Fig 19. The $R^{2}$-correlation coefficient was excellent again, meaning that Eq. 7 is suitable to model closure of ellipsoidal voids.

For each ellipsoid, three coefficients $B^{\overrightarrow{u_{i}}}$ (and $C^{\overrightarrow{u_{i}}}$ ) were obtained, respectively along their three principal directions $i=\{1,2,3\}$. The values obtained for $B^{\overrightarrow{u_{i}}}$ (and $C^{\overrightarrow{u_{i}}}$ ) are plotted in Fig. 20 versus a geometry parameter defined as

$$
\gamma_{i}=\frac{\sqrt[3]{V_{0}}}{r_{i}}
$$

This parameter represents an apparent geometry parameter according to the $\overrightarrow{u_{i}}$ direction. It is a non-dimension parameter that considers tridimensional features of the geometry with respect to each principal direction. For example, in the case of the first principal direction $\overrightarrow{u_{1}}$, the parameter can be expressed as:

$$
\gamma_{1}=\frac{\sqrt[3]{V_{0}}}{r_{1}}=\frac{\sqrt[3]{\frac{4 \pi}{3} r_{1} r_{2} r_{3}}}{r_{1}}=\sqrt[3]{\frac{\frac{4 \pi}{3} r_{1} r_{2} r_{3}}{r_{1}^{3}}}=\sqrt[3]{\frac{4 \pi}{3} \frac{r_{2}}{r_{1}}} \frac{r_{3}}{r_{1}} .
$$

The $\gamma_{1}$ parameter is proportional to the cubic root of the product of aspect ratios. In this manner, this parameter contains the information relative to the void's tridimensional geometry. This parameter was chosen as it provided a bijective relation with the $B^{\overrightarrow{u_{i}}}$ and $C^{\overrightarrow{u_{i}}}$ coefficients, regardless of the value of $i$. 
As expected, due to axisymmetrical features, it was verified that for prolate ellipsoids, $B^{\overrightarrow{u_{1}}}=$ $B^{\overrightarrow{u_{2}}}$ and $C^{\overrightarrow{u_{1}}}=C^{\overrightarrow{u_{2}}}$. Regarding oblate ellipsoids it was found that $B^{\overrightarrow{u_{2}}}=B^{\overrightarrow{u_{3}}}$ and $C^{\overrightarrow{u_{2}}}=C^{\overrightarrow{u_{3}}}$. Finally, for the spherical void $B^{\overrightarrow{u_{1}}}=B^{\overrightarrow{u_{2}}}=B^{\overrightarrow{u_{3}}}$ and $C^{\overrightarrow{u_{1}}}=C^{\overrightarrow{u_{2}}}=C^{\overrightarrow{u_{3}}}$.

\begin{tabular}{|llllll|}
\hline & $\left(p_{1}, p_{2}\right)=(1.0,0.0)$ & & $\left(p_{1}, p_{2}\right)=(0.0,1.0)$ & & $\left(p_{1}, p_{2}\right)=(0.0,0.0)$ \\
$\bullet$ & Sphere $\left(R^{2}=0.9991\right)$ & $\square$ & Sphere $\left(R^{2}=0.9991\right)$ & $\star$ & Sphere $\left(R^{2}=0.9991\right)$ \\
$\bullet$ & Ell-A $\left(R^{2}=0.9968\right)$ & $\square$ & Ell-A $\left(R^{2}=0.9968\right)$ & $\star$ & Ell-A $\left(R^{2}=0.9982\right)$ \\
$\bullet$ & Ell-B $\left(R^{2}=0.9935\right)$ & $\square$ & Ell-B $\left(R^{2}=0.9930\right)$ & $\star$ & Ell-B $\left(R^{2}=0.9847\right)$ \\
$\square$ & Ell-C $\left(R^{2}=0.9981\right)$ & $\square$ & Ell-C $\left(R^{2}=0.9971\right)$ & $\star$ & Ell-C $\left(R^{2}=0.9970\right)$ \\
$\bullet$ & Ell-D $\left(R^{2}=0.9909\right)$ & $\square$ & Ell-D $\left(R^{2}=0.9984\right)$ & $\star$ & Ell-D $\left(R^{2}=0.9984\right)$ \\
$\bullet$ & Ell-E $\left(R^{2}=0.9982\right)$ & $\square$ & Ell-E $\left(R^{2}=0.9960\right)$ & $\star$ & Ell-E $\left(R^{2}=0.9989\right)$ \\
$\bullet$ & Ell-F $\left(R^{2}=0.9934\right)$ & $\square$ & Ell-F $\left(R^{2}=0.9914\right)$ & $\star$ & Ell-F $\left(R^{2}=0.9996\right)$ \\
\hline
\end{tabular}
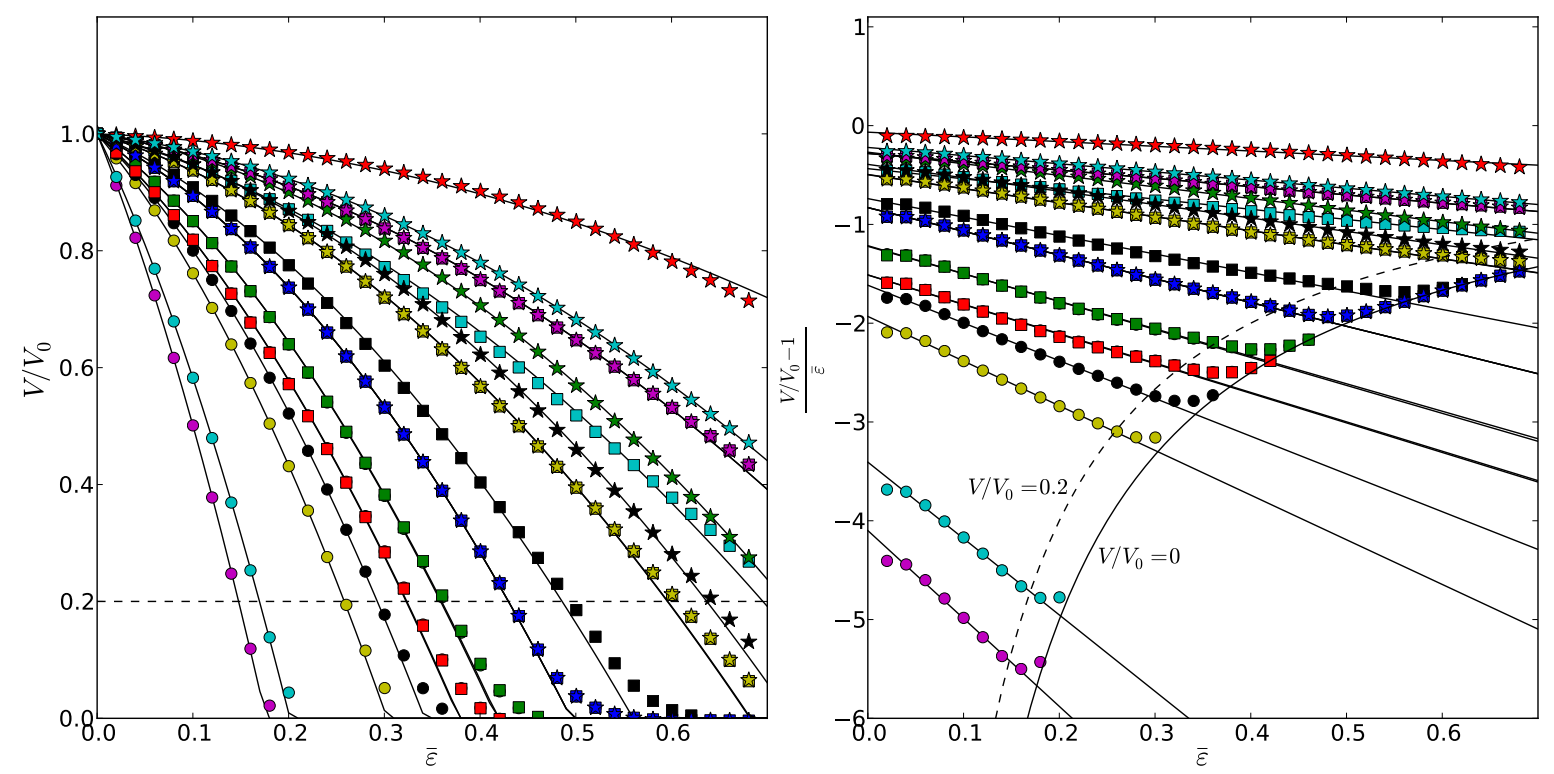

Figure 19: Curve fitting (with $R^{2}$-correlation factor) performed using RVE results for various geometries and orientations (given in Fig. 9), by linear regression using the linearized expression (Eq. 8).

On Fig. 20, all cases are presented on the same plot in order to establish a unique relationship $b\left(\gamma_{i}\right)$ (and $c\left(\gamma_{i}\right)$ ) between void closure and the apparent geometry parameter. This is done because, for a given apparent geometry parameter, the same mechanisms are involved whether the apparent geometry corresponds to the first, the second or the third principal direction. Eq. 9 thus becomes:

$$
\begin{aligned}
& B=b\left(\gamma_{1}\right) p_{1}+b\left(\gamma_{2}\right) p_{2}+b\left(\gamma_{3}\right) p_{3}, \\
& C=c\left(\gamma_{1}\right) p_{1}+c\left(\gamma_{2}\right) p_{2}+c\left(\gamma_{3}\right) p_{3} .
\end{aligned}
$$

From Fig. 20, a bijective monotonously decreasing evolution was observed ${ }^{2}$. A quadratic polynomial function is proposed such that:

$$
\begin{aligned}
& b\left(\gamma_{i}\right)=b_{0}+b_{1} \gamma_{i}+b_{2} \gamma_{i}^{2}, \\
& c\left(\gamma_{i}\right)=c_{0}+c_{1} \gamma_{i}+c_{2} \gamma_{i}^{2} .
\end{aligned}
$$

\footnotetext{
${ }^{2}$ The values in $y$-abscissa were intentionally erased for confidentiality reasons.
} 
The values plotted in Fig. 20 were used to identify coefficients $b_{0}, b_{1}$ and $b_{2}$ (and $c_{0}, c_{1}$ and $c_{2}$ ) using polynomial regression analysis. The $R^{2}$-correlation factor is very good again.

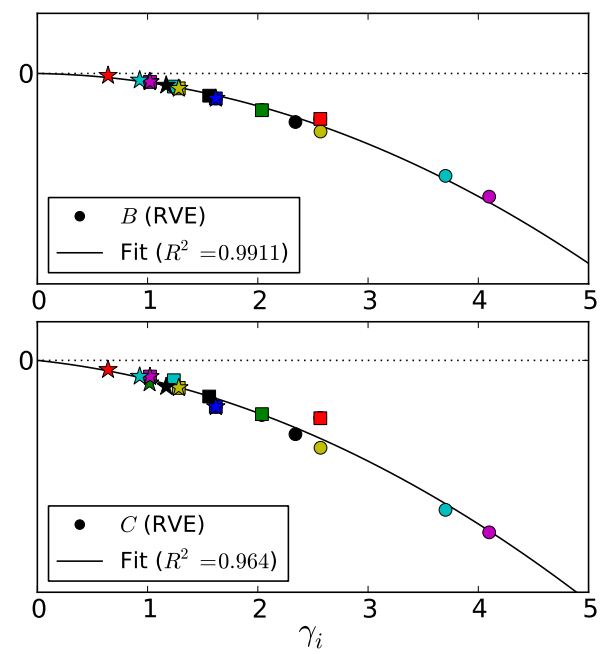

Figure 20: Influence of the geometry parameter $\gamma_{i}$ on $B$ and $C$ coefficients, obtained from fitting for all cases given in Fig. 19 (common legend).

\subsection{Dependence to stress triaxiality ratio}

Eq. 13 was obtained for the particular value $T_{X}=-1 / 3$, i.e. under uniaxial compression (using the boundary conditions $\sigma_{x}=\sigma_{y}=0$ ).

In order to quantitatively assess the influence of stress triaxiality ratio and to extend Eq. 13, the analysis was repeated under various stress triaxiality ratios over the range $T_{X}=[-1,0]$. The evolution of $b\left(\gamma_{i}\right)$ and $c\left(\gamma_{i}\right)$ (i.e. the dependence to geometry) is plotted ${ }^{3}$ for various stress triaxiality ratios in Fig. 21. The curves exhibit a rather linear dependence to the geometry parameter $\gamma_{i}$ for low compressive stress states. Non-linearity occurs when decreasing the value of $T_{X}$.

In Fig. 21, it can be seen that the fitting accuracy also decreases for compressive stress triaxiality ratios. The case that is plotted using a red square exhibits rather large discrepancy. It corresponds to Ellipsoid B when it is deformed perpendicularly to its longest direction (see Fig. 8).

The coefficients $b_{i}$ and $c_{i}(i \in\{0,1,2\})$ were obtained using polynomial regression analysis for each case and are plotted ${ }^{3}$ in Fig. 22.

A linear dependence is observed for the $b_{i}$ coefficients over the whole range of $T_{X}$. For the $c_{i}$ coefficients, a linear dependence is observed as well on the range $-0.4 \leq T_{X} \leq 0$, and a quadratic dependence is observed on the range $-1 \leq T_{X} \leq-0.4$. The regression was therefore split and two batches of calibration constants were obtained, one for each range of $T_{X}$. The good values of $R^{2}$-correlation coefficients indicate that the chosen analytical functions are suitable to model these dependences.

\footnotetext{
${ }^{3}$ The values in $y$-abscissa were intentionally erased for confidentiality reasons.
} 

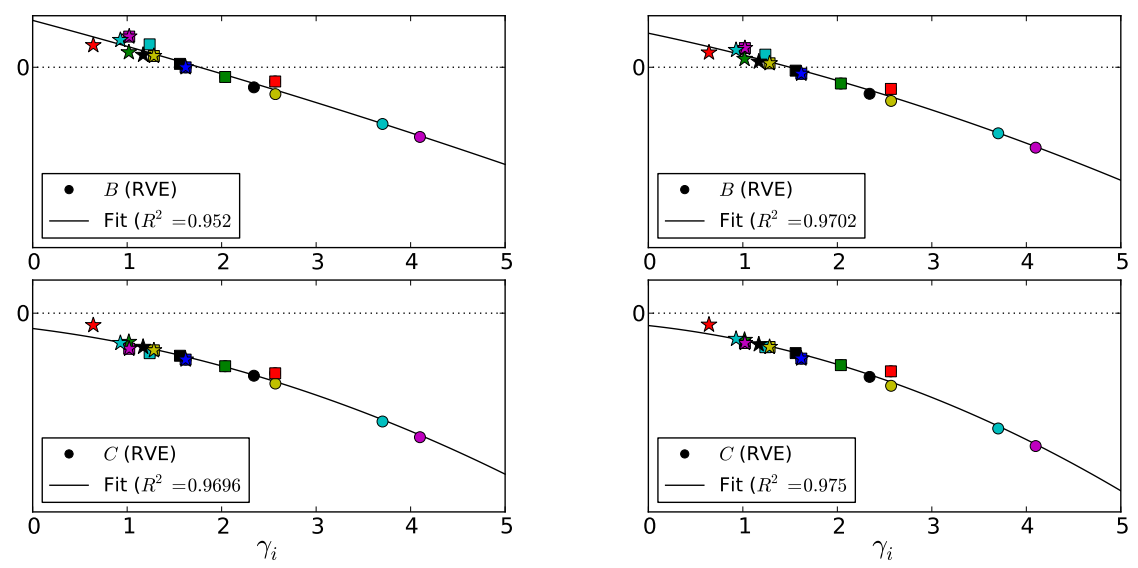

(a) $T_{X}=-0.04$

(b) $T_{X}=-0.13$
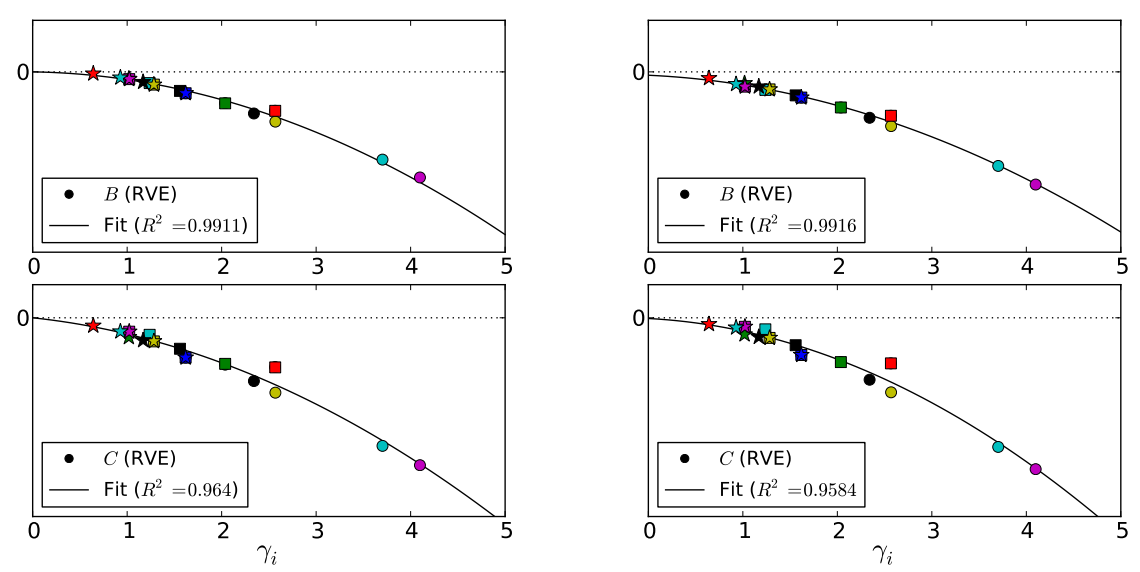

(c) $T_{X}=-0.33$

(d) $T_{X}=-0.39$
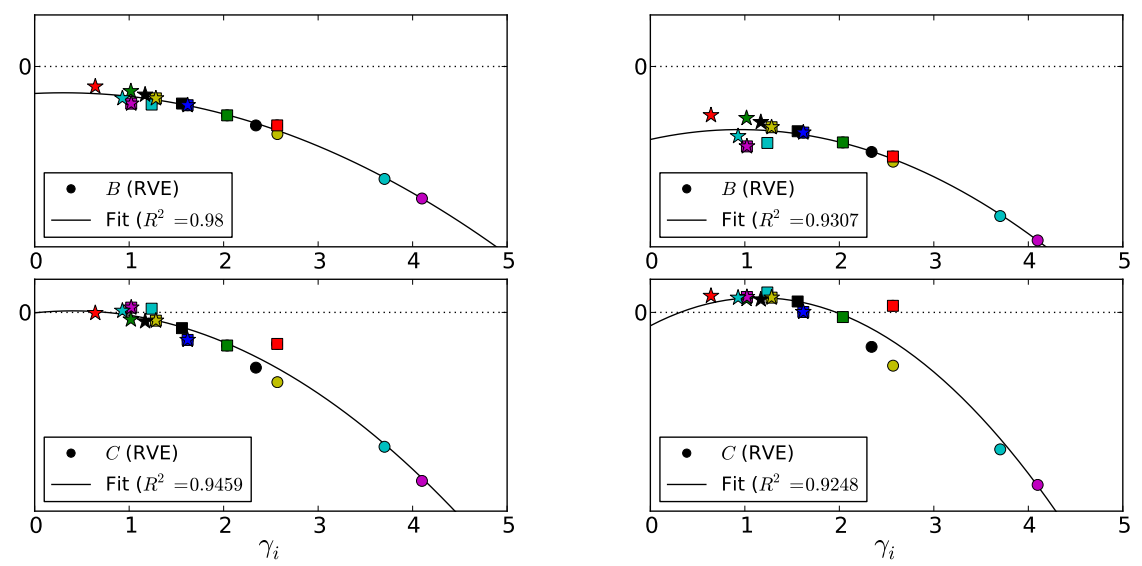

(e) $T_{X}=-0.57$

(f) $T_{X}=-0.94$

Figure 21: Comparison, for several values of stress triaxiality ratio $T_{X}$, of the influence of the geometry parameter $\gamma_{i}$ on $B$ and $C$ coefficients, obtained from curve fitting (see legend for the dots in Fig. 19). 

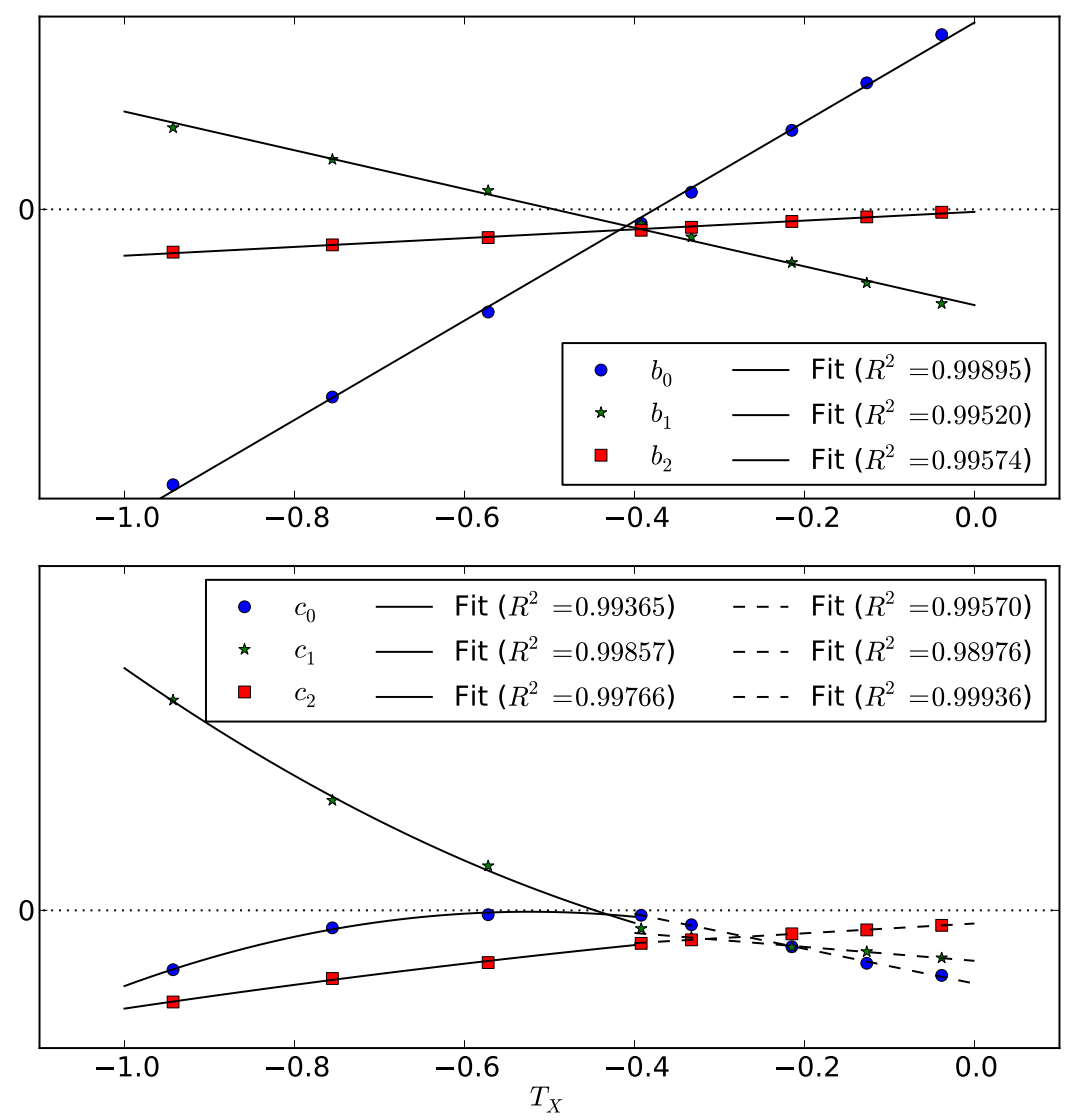

Figure 22: Influence of stress triaxiality on the $b_{i}$ and $c_{i}$ coefficients $(i \in\{0,1,2\})$ from Eq. 13 .

The final dependence to $\gamma_{i}$ and to stress triaxiality ratio $T_{X}$ can be written as:

$$
\begin{array}{lll}
b\left(\gamma_{i}\right)=b_{00}+b_{10} \gamma_{i}+b_{20} \gamma_{i}^{2} & +T_{X}\left(b_{01}+b_{11} \gamma_{i}+b_{21} \gamma_{i}^{2}\right), \\
c\left(\gamma_{i}\right)=c_{00}+c_{10} \gamma_{i}+c_{20} \gamma_{i}^{2} & +T_{X}\left(c_{01}+c_{11} \gamma_{i}+c_{21} \gamma_{i}^{2}\right)+T_{X}^{2} & \left(c_{02}+c_{12} \gamma_{i}+c_{22} \gamma_{i}^{2}\right)
\end{array}
$$

where $b_{i j}$ and $c_{i j}$ are calibration constants.

The coefficients $B$ and $C$ are called closure coefficients. In fact, $B$ can be seen as the initial closure coefficient, providing the initial slope of the void volume evolution at $\varepsilon=0$. The coefficient $C$ can be seen as a deformation-dependent coefficient, translating the change of shape during deformation. This is in good agreement with the results of Lee and Mear (1994). The authors pointed out the necessity to consider the change of shape of a void during deformation.

In the present work, the coefficient is calibrated under constant loadings. The value of $C$ thus contains the information of the change of shape during a uniform loading.

For a better prediction, the current evolution of the void must be known throughout the deformation. For a single pass process, this assumption is correct as the material is generally loaded along one main axis.

\subsection{Final expression of the prediction model}

Finally, the crossed dependences to orientation, to geometry and to stress triaxiality ratio can be summarized in a single expression: 


$$
\frac{V}{V_{0}}=1+B \bar{\varepsilon}+C \bar{\varepsilon}^{2} \quad \text { with }\left\{\begin{array}{l}
B=\sum_{i=1}^{3} \sum_{j=0}^{2} \sum_{k=0}^{1} b_{j k}\left(T_{X}\right)^{k}\left(\gamma_{i}\right)^{j} p_{i} \\
C=\sum_{i=1}^{3} \sum_{j=0}^{2} \sum_{k=0}^{2} c_{j k}\left(T_{X}\right)^{k}\left(\gamma_{i}\right)^{j} p_{i}
\end{array}\right.
$$

\section{Validation of the prediction model}

In order to verify the reliability of the proposed model, predicted evolutions are compared to the void volume evolutions that were measured for several ellipsoidal voids, under various mechanical conditions. Four morphology-equivalent ellipsoids were obtained from tridimensional observations of real voids, such as presented in previous work (Saby et al., 2013). The RVEs containing these ellipsoidal voids are illustrated in Fig. 23. Various stress triaxiality values are prescribed on the range $T_{X}=[-1,0]$.

\begin{tabular}{lcccccc}
\hline & \multicolumn{3}{c}{ Dimensions $(\mathrm{mm})$} & \multicolumn{3}{c}{ Orientation } \\
& $r_{1}$ & $r_{2}$ & $r_{3}$ & $p_{1}$ & $p_{2}$ & $p_{3}$ \\
\hline Ellipsoid A1 & 1.35 & 1.62 & 2.36 & 0.97 & 0.01 & 0.02 \\
Ellipsoid A2 & 1.20 & 1.42 & 2.10 & 0.67 & 0.10 & 0.23 \\
Ellipsoid A3 & 1.34 & 1.63 & 3.17 & 0.88 & 0.21 & 0.01 \\
Ellipsoid A4 & 0.47 & 1.61 & 3.97 & 0.20 & 0.22 & 0.58 \\
\hline
\end{tabular}

Table 1: Geometrical features of the ellipsoids used for the validation of the calibration.

Void volume evolution is thus plotted for each ellipsoid in Fig. 24. For each plot, the predicted volume evolution is plotted as well. It is computed using Eq. 15, according to the geometrical features given in Table. 1 and the prescribed stress triaxiality ratio $T_{X}$.

The objective here is to assess the ability of the model to predict the behaviour of ellipsoids. This is directly related to the efficiency of the chosen model, as well as the calibration step.

The aspect ratios of ellipsoid A1 can be obtained from its dimensions and are $\left(r_{3} / r_{1}\right)=1.75$ and $\left(r_{2} / r_{1}\right)=1.20$. The orientation parameter $p_{1}$ is close to unity and $p_{2} \approx p_{3} \approx 0$. These values of orientation indicate that the principal vector of the void $\overrightarrow{u_{1}}$ is colinear with the main deformation direction $\overrightarrow{e_{1}}$. In other words, the void is mainly deformed along its smallest dimension $r_{1}$. As a consequence, according to Eq. 12, the closure coefficients $\mathrm{b}$ and c are respectively equal to $b\left(\gamma_{1}\right)$ and $c\left(\gamma_{1}\right)$.

The $L^{2}$-norm error was computed between the model and the result from RVE simulation. For all tested values of stress triaxiality ratio, the error remains lower than 5\%. In the case of Ellipsoid A1, the model is capable of predicting its void volume with very good accuracy.

The aspect ratios of ellipsoid A2 are $\left(r_{3} / r_{1}\right)=1.75$ and $\left(r_{2} / r_{1}\right)=1.18$. Note that, by chance, the values are roughly identical to the ones of ellipsoid A1. Regarding its orientation, ellipsoid A2 is slightly tilted compared to ellipsoid A1. As a consequence, the values of closure coefficients $B$ (respectively coefficients $C$ ) are given by a linear combination of $b\left(\gamma_{1}\right), b\left(\gamma_{2}\right)$ and $b\left(\gamma_{3}\right)$ (respectively $c\left(\gamma_{1}\right), c\left(\gamma_{2}\right)$ and $\left.c\left(\gamma_{3}\right)\right)$, according to Eq. 12 .

For the lowest triaxiality ratios, the prediction is excellent, with a $L^{2}$-norm error about $3 \%$. When increasing the value of stress triaxiality, the prediction slightly underestimates void closure, 


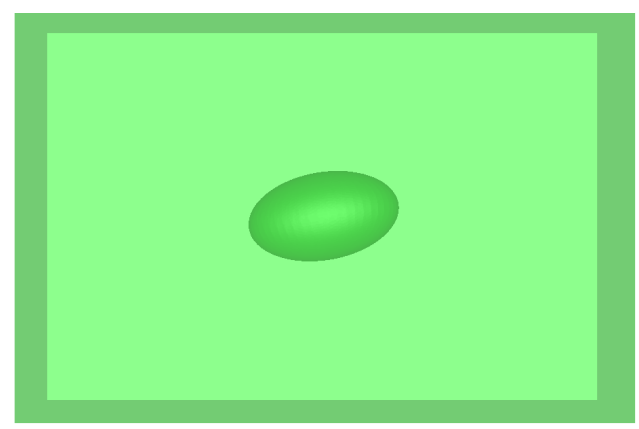

(a) Ellipsoid A1

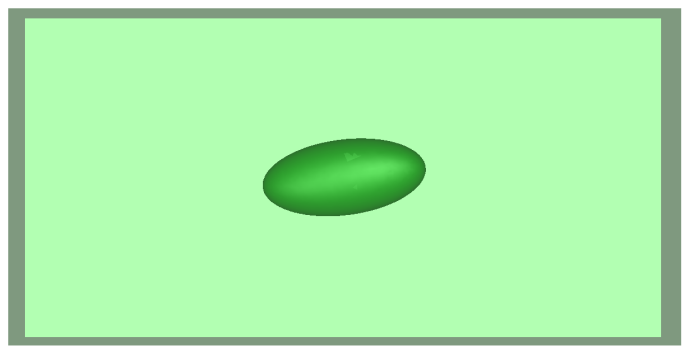

(c) Ellipsoid A3

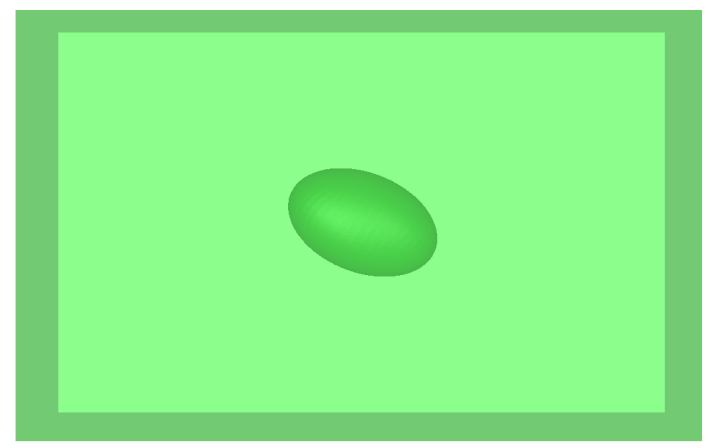

(b) Ellipsoid A2

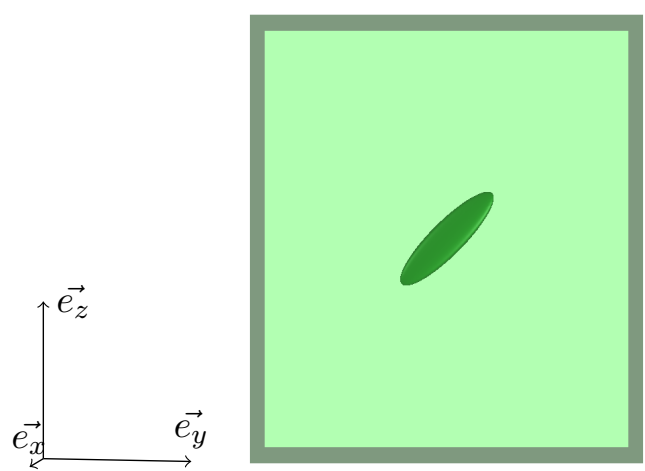

(d) Ellipsoid A4

Figure 23: Initial RVEs containing morphology-equivalent ellipsoids.

the $L^{2}$-norm error remaining lower than $9 \%$ over the whole range of $T_{X}$. The obtained error is mainly due to the fact that the closure rates are relatively small for low compressive states. When looking at Fig. 21, the relative error that is made by regression for the value of $B$ is higher for the value $T_{X}=-0.04$ than for the value $T_{X}=-0.94$, due to the fact that the values are close to zero when $T_{X}$ tends to zero. As a consequence, the error made on the value of $B$ is visible on the initial slope of the volume evolutions in Fig. 24.

In Fig. 25, the void volume predictions obtained from the model of Zhang et al. (2009) and from the STB model are plotted for ellipsoid A2 and the $L^{2}$-norm error is given. Note that the use of the Zhang model requires the definition of a material parameter $m^{*}$. The authors provide calibration coefficients for a discrete series of values $m^{*}=\{0.01,0.1,0.2,1 / 3,0.5,1\}$. Since the model was theoretically developed for viscoplastic materials without strain hardening, the value of $m^{*}$ may not be strictly defined in the present case, since a viscoplastic material containing strain hardening was used in the present FE simulations. Among all possible values of $m^{*}$, the value that provided the lowest error between the Zhang model and the FE simulations was retained $\left(m^{*}=0.2\right)$ and the results are illustrated in Fig. 25a.

In the Zhang model, the initial slope at $\bar{\varepsilon}=0$ shows good agreement with FE-data. However, this agreement decreases when deformation increases. Using the Zhang model, the prediction leads to a $L^{2}$-norm error on the range [7-17]\%, while it is on the range [3-9]\% for the new proposed model. The error is mainly due to the analytical function that is expressed to model the void volume evolution during deformation. 


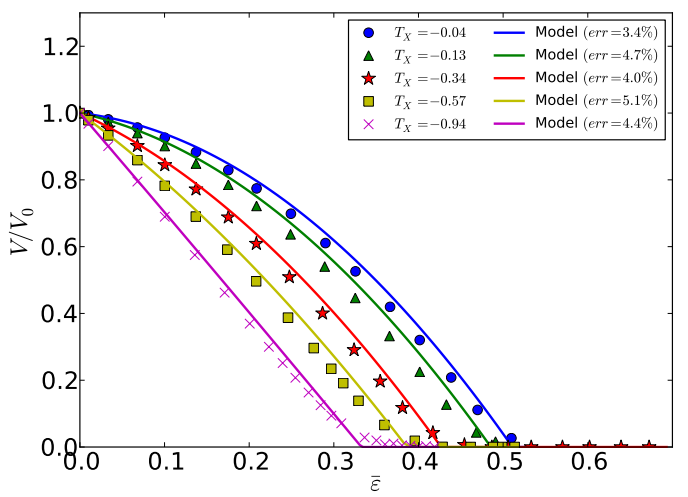

(a) Ellipsoid A1

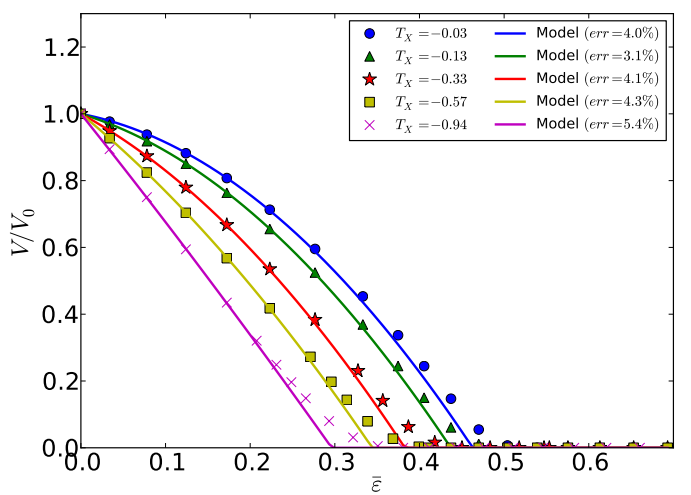

(c) Ellipsoid A3

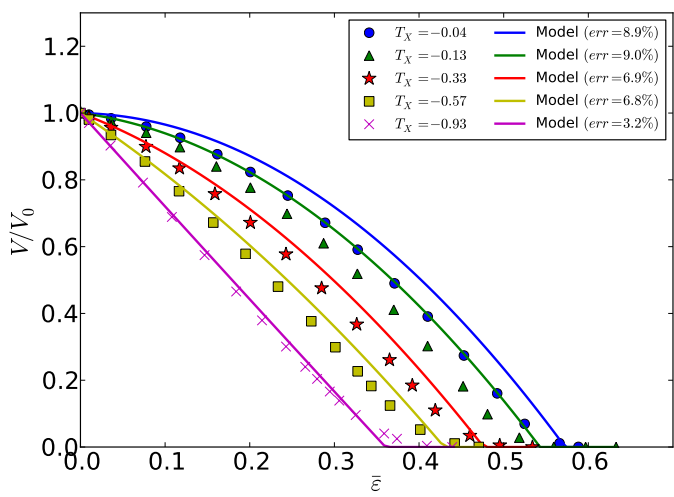

(b) Ellipsoid A2

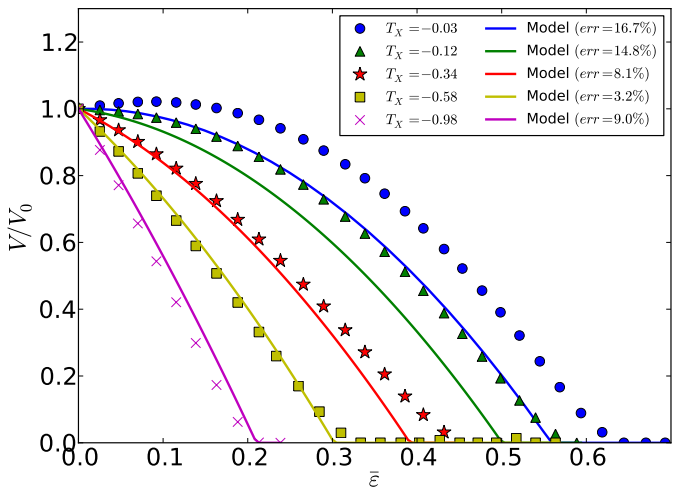

(d) Ellipsoid A4

Figure 24: Illustration of the $L^{2}$-norm error made by the prediction model on the actual closure of an ellipsoidal void.

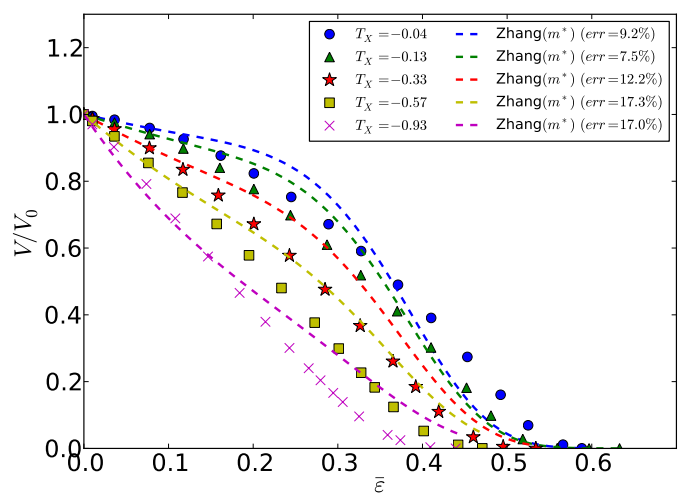

(a) Model of Zhang et al. (2009), using a material parameter $m^{*}=0.2$

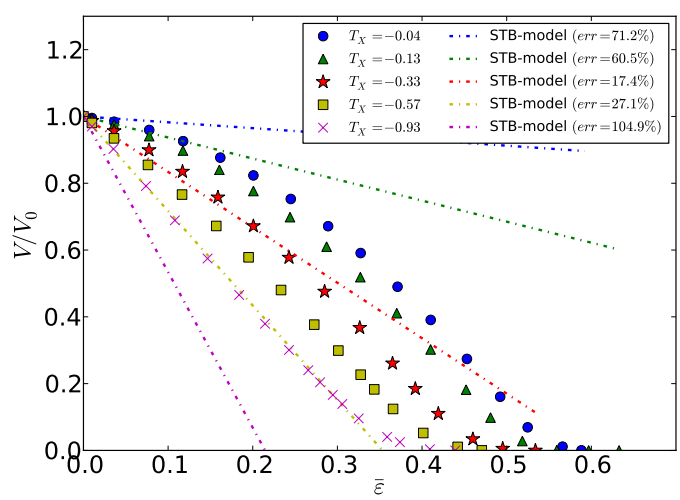

(b) STB-model

Figure 25: Illustration of the $L^{2}$-norm error made by existing models for the prediction of Ellipsoid A2. 
In the case of the STB model, the analytical function that is used is a linear expression. According to the FE-data, this linear expression is clearly inadequate when deformation increases. The resulting $L^{2}$-norm error rises over $100 \%$.

The aspect ratios of ellipsoid A3 are $\left(r_{3} / r_{1}\right)=2.37$ and $\left(r_{2} / r_{1}\right)=1.22$. In this case, the second aspect ratio is relatively similar to the previous ones as well. However, the first aspect ratio is larger, indicating that the void is more elongated along its third principal direction. Its orientation parameters exhibit the particular value of $p_{3} \approx 0$, meaning that the third principal direction is perpendicular to the $z$-axis (the deformation axis). The behaviour of the void is thus driven by a combination of the closure coefficients $b\left(\gamma_{1}\right)$ and $b\left(\gamma_{2}\right)$ (and $c\left(\gamma_{1}\right)$ and $c\left(\gamma_{2}\right)$ ), only.

The $L^{2}$-norm error remains lower than $5 \%$. The accuracy of the prediction for ellipsoid A3 is comparable to the one obtained for ellipsoid A1.

The aspect ratios of ellipsoid A4 are $\left(r_{3} / r_{1}\right)=8.45$ and $\left(r_{2} / r_{1}\right)=3.43$. The orientation parameters indicate that the void does not show any particular orientation.

Regarding Eq. 14, the apparent geometrical parameters $\gamma_{1}=4.95$ and $\gamma_{3}=0.59$ of ellipsoid A4 are situated at the boundaries of the range of tested values for the calibration (see Fig. 21). The accuracy of the regression is therefore lower for such values, and the resulting error is visible on the final prediction of void volume in Fig. 24d. This case can therefore be considered as an extreme case for the validation of the model.

The same comment can be made regarding the fact that the maximum discrepancy is obtained for low compressive values of stress triaxiality ratio. The $L^{2}{ }^{-}$norm error rises to $17 \%$ in the case $T_{X}=0$. For more compressive triaxiality ratios, the error remains rather low, with the best prediction obtained for the value $T_{X}=-0.58$, with an error of about $3 \%$.

\section{Conclusions}

Based on a wide campaign of FE simulations at the RVE-scale, the significant influence of geometry and orientation of a void on its closure behaviour was demonstrated. The influence of stress state (using the stress triaxiality ratio on the range $[-1,0]$ ) was also verified. These crossed influences were quantified and a new prediction model for void closure was proposed.

Four ellipsoids were used as validation cases. Their geometry and orientation was obtained from tridimensional observations of real industrial voids. The model provides very good prediction for all cases under several stress states. The prediction error is significantly lower than the error obtained from the models from literature (based on spherical voids).

The chosen analytical expression is thus suitable for an accurate prediction of void closure for an ellipsoid of any geometry, in any orientation and under any stress triaxiality ratio. This model can easily be implemented as a post-processing subroutine and linked with process simulation. Process design can consequently be performed with greater accuracy. This is the object of a forthcoming publication.

Further developments would also be necessary to keep track of void geometry during deformation. This additionnal information would show great interest regarding multipass processes.

\section{Acknowledgments}

The authors would like to gratefully thank TIMET Savoie, as well as Aubert\&Duval, Ascometal, Areva NP, Industeel and Constellium for funding this research. 
Banaszek, G., Stefanik, A., 2006. Theoretical and laboratory modelling of the closure of metallurgical defects during forming of a forging. Journal of Materials Processing Technology 177 (1-3), 238-242.

URL http: //www. sciencedirect. com/science/article/B6TG J-4K4PSMM- G/2/1ddc0f5e0d8c0f 176b98f9d21e0de0d2

Chen, J., Chandrashekhara, K., Mahimkar, C., Lekakh, S. N., Richards, V. L., 2012. Study of void closure in hot radial forging process using 3D nonlinear finite element analysis. The International Journal of Advanced Manufacturing Technology 62 (9-12), 1001-1011. URL http://dx.doi.org/10.1007/s00170-011-3876-3

Dudra, S. P., Im, Y.-T., 1990. Analysis of void closure in open-die forging. International Journal of Machine Tools and Manufacture 30 (1), 65-75.

URL http://www.scopus.com/inward/record.url?eid=2-s2.0-0025068159\&partnerID=40\&md5= f2a163baad983e60164d2eb834a7fc94

Duva, J. M., Hutchinson, J. W., 1984. Constitutive potentials for dilutely voided nonlinear materials. Mechanics of Materials 3 (1), 41-54.

URL http: //www. sciencedirect. com/science/article/B6TX6-47Y9N8C-3G/2/b3859a4ce80ab8b30c4c7bd9adfedf 20

FORGE, 2011. 2011 ${ }^{\circledR}$. Transvalor S.A., 694, av. Donat, 06255 Mougins Cedex, France.

He, M. Y., Hutchinson, J. W., 1981. The penny-shaped crack and the plane strain crack in an infinite body of power-law material. Journal of Applied Mechanics 48, 830-840.

URL http://www. seas .harvard.edu/hutchinson/papers/360.pdf

Kakimoto, H., Arikawa, T., Takahashi, Y., Tanaka, T., Imaida, Y., 2010. Development of forging process design to close internal voids. Journal of Materials Processing Technology 210 (3), 415-422.

URL http://ww. scopus.com/inward/record.url?eid=2-s2.0-72149099080\&partnerID=40\&md5= cf6daf9d13e23b77817d1ff43b5639de

Lee, B. J., Mear, M. E., 1994. Studies of the growth and collapse of voids in viscous solids. Journal of Engineering Materials and Technology, Transactions of the ASME 116 (3), 348-358.

URL http://www.scopus.com/inward/record.url?eid=2-s2.0-0028460868\&partnerID=40\&md5= fc5951a1928a5bf58a1d43675898eb83

Nakasaki, M., Takasu, I., Utsunomiya, H., 2006. Application of hydrostatic integration parameter for free-forging and rolling. Journal of Materials Processing Technology 177 (1-3), 521-524.

URL http://www.sciencedirect. com/science/article/B6TG J-4K9C58P-3/2/d8acda428804338f74c8580b2b5da19d

Överstam, H., Jarl, M., 2004. Fem-simulation of drawing out in open die forging. Steel Research International 75 (12), $812-817$.

URL http://cat.inist.fr/?aModele=afficheN\&cpsidt $=16340484$

Park, C. Y., Yang, D. Y., 1996. A study of void crushing in large forgings I: Bonding mechanism and estimation model for bonding efficiency. Journal of Materials Processing Technology 57 (1-2), 129-140.

URL http://ww. scopus. com/inward/record.url? eid=2-s2.0-0030086410\&partnerID=40\&md5= caf9071f905d25d15fb02256ba81f42d

Pietrzyk, M., Kawalla, R., Pircher, H., 1995. Simulation of the behaviour of voids in steel plates during hot rolling. Steel Research 66 (12), 526-529.

URL http://ww. scopus.com/inward/record.url?eid=2-s2.0-0029485195\&partnerID=40\&md5= 2b91d770b5950c13bece9906ce097788

Ragab, A. R., 2004. Application of an extended void growth model with strain hardening and void shape evolution to ductile fracture under axisymmetric tension. Engineering Fracture Mechanics 71 (11), 1515-1534. URL http://www. sciencedirect. com/science/article/B6V2R-49PRFB4-5/2/acf52c9246e963b93481716b5c177004

Saby, M., 2013. Understanding and modeling of void closure mechanisms in hot metal forming processes. Ph.D. thesis, Ecole Nationale Supérieure des Mines de Paris.

Saby, M., Bernacki, M., Roux, E., Bouchard, P.-O., 2013. Three-dimensional analysis of real void closure at the meso-scale during hot metal forming processes. Computational Materials Science 77, 194-201.

URL http://ww. sciencedirect.com/science/article/pii/S0927025613002358

Ståhlberg, U., 1986. Influence of spread and stress on the closure of a central longitudinal hole in the hot rolling of steel. Journal of Mechanical Working Technology 13 (1), 65-81.

URL http://www.sciencedirect.com/science/article/pii/0378380486900434

Ståhlberg, U., Keife, H., 1992. A study of hole closure in hot rolling as influenced by forced cooling. Journal of Materials Processing Technology 30 (1), 131 - 135.

URL http://www.sciencedirect.com/science/article/pii/092401369290043R

Ståhlberg, U., Keife, H., Lundberg, M., Melander, A., 1980. A study of void closure during plastic deformation. Journal of Mechanical Working Technology 4 (1), 51-63. 
URL

http: //www. scopus. com/inward/record.url?eid=2-s2.0-0010584536\&partnerID=40\&md5= 1d0ae86a102a695ddf640b01e876544c

Tanaka, M., Ono, S., Tsuneno, M., 1986. Factors contributing to crushing of voids during forging. Journal of JSTP 27 (306), 927-934, (translated from Japanese).

Tomlison, A., Met, A., Dstringer, J., 1958. La suppression des cavites internes dans les pieces de forge par refoulement. Journal of the Iron and Steel Institute, 209-218.

Wallerö, A., 1985. Closing of a central longitudinal pore in hot rolling. Journal of Mechanical Working Technology 12 (2), 233-242.

URL http: //www.sciencedirect.com/science/article/B756R-481DT7H-BB/2/b5b916dda84d2e32d5ce84c117f4b895

Zhang, X.-X., Cui, Z.-S., 2009. Theoretical study of void closure in nonlinear plastic materials. Applied Mathematics and Mechanics-english Edition 30 (5), 631-642.

Zhang, X.-X., Cui, Z.-S., Chen, W., Li, Y., 2009. A criterion for void closure in large ingots during hot forging. Journal of Materials Processing Technology 209 (4), 1950-1959.

URL http://ww. scopus.com/inward/record.url?eid=2-s2.0-58249143493\&partnerID=40\&md5= d902043a690364a783789fd48df c0d5c 